\title{
Resting-state EEG Coupling Analysis of Amnestic Mild Cognitive Impairment with Type 2 Diabetes Mellitus by Using Permutation Conditional Mutual Information
}

\author{
Dong Wen ${ }^{\mathrm{a}, \mathrm{b}}$, Zhijie Bian ${ }^{\mathrm{c}}$, Qiuli $\mathrm{Li}^{\mathrm{d}}$, Lei Wang ${ }^{\mathrm{d}^{*}}$, Chengbiao Lu ${ }^{\mathrm{c}}$, Xiaoli $\mathrm{Li}^{\mathrm{c}^{*}}$ \\ ${ }^{a}$ School of Information Science and Engineering, Yanshan University, Qinhuangdao, China \\ ${ }^{\mathrm{b}}$ The Key Laboratory for Computer Virtual Technology and System Integration of Hebei Province, Yanshan \\ University, Qinhuangdao, China \\ ${ }^{\mathrm{c}}$ School of Electrical Engineering, Yanshan University, Qinhuangdao, China \\ ${ }^{\mathrm{d}}$ Department of Neurology, The Second Artillery General Hospital of PLA, Beijing, China
}

Two corresponding authors:

Prof. Xiaoli Li (xlli@ysu.edu.cn) and Dr Lei Wang (wencangdong@163.com)

* Major corresponding information:

Xiaoli Li (xlli@ysu.edu.cn)

438, Hebei Avenue, Qinhuangdao City, 066004, Hebei Province, P.R. China

Tel.: +8613784062860

Fax: +863358387556 


\section{ABSTRACT}

Objective: This study was meant to explore whether the coupling strength and direction of resting-state electroencephalogram (rsEEG) could be used as an indicator to distinguish the patients of Type 2 Diabetes Mellitus (T2DM) with or without amnestic Mild Cognitive Impairment (aMCI).

Methods: Permutation conditional mutual information (PCMI) was used to calculate the coupling strength and direction of rsEEG signals between different brain areas of $19 \mathrm{aMCI}$ and 20 normal control (NC) with T2DM on 7 frequency bands: Delta, Theta, Alpha1, Alpha2, Beta1, Beta2 and Gamma. The difference in coupling strength or direction of rsEEG between two groups was calculated. The correlation between coupling strength or direction of rsEEG and score of different neuropsychology scales were also calculated.

Results: We have demonstrated that PCMI can calculate effectively the coupling strength and directionality of EEG signals between different brain regions. The significant difference in coupling strength and directionality of EEG signals was found between the patients of aMCI and $\mathrm{NC}$ with $\mathrm{T} 2 \mathrm{DM}$ on different brain regions. There also existed significant correlation between sex or age and coupling strength or coupling directionality of EEG signals between a few different brain regions from all subjects.

Conclusions: The coupling strength or directionality of EEG signals calculated by PCMI are significantly different between aMCI and NC with T2DM.

Significance: These results showed that the coupling strength or directionality of EEG signals calculated by PCMI might be used as a biomarker in distinguishing the aMCI from NC with T2DM.

Keywords: Resting-state EEG; coupling; amnestic mild cognitive impairment; T2DM; permutation conditional mutual information. 


\section{Introduction}

Diabetes is a kind of metabolic diseases characterized by high blood sugar due to insufficient secretion of insulin or cellular resistance to insulin (Shoback, 2011). Diabetes affected cognitive function and increased the risk of dementia (Gispen et al. , 2000, Strachan et al. , 2011, Bian et al. , 2014). Type 2 diabetes mellitus (T2DM) is a late-onset, most common type of diabetes (Kumar, 2005). Cognitive impairment such as learning and memory deficiency were documented in T2DM (Peila et al. , 2002, Meihua Qu, 2012, Huerta et al. , 2013, Moran et al. , 2013, Roberts et al. , 2014). The T2DM may be associated with increased risk of aMCI (Ganguli et al., 2004, Busse et al., 2006, Yaffe et al., 2006, Fischer et al., 2007, Luchsinger et al. , 2007, Toro et al. , 2009, Shimada et al. , 2010, Xu et al., 2010, Strachan et al. , 2011, Tuma, 2012, Roberts et al., 2014, Bian et al., 2014, Zhang, et al., 2014).. Therefore, it is critical to explore effective methods to detect the aMCI of T2DM patients for the early interventions to these patients.

The resting-state EEG (rsEEG) underlies brain network activity (Steriade, 2006) and can be used for neurological evolution (Rossini et al. , 2007, Schmidt et al. , 2013). Recent studies have shown that rsEEG rhythms maybe a promising approach to diagnose MCI subjects (Dauwels et al. , 2010b, Knyazeva et al. , 2013, Babiloni et al. , 2014, Dong Wen, 2014). There were also several studies about the cognitive function of T2DM using EEG signals (Gerald Cooray, 2008, Cooray et al. , 2011, Baskaran et al. , 2012, Bian et al. , 2014).

Due to the nature of complex characteristics in EEG signals, many methods were used to analyze the EEG signal from different perspectives (Gerald Cooray, 2008, Dauwels et al. , 2010a, Cooray et al. , 2011, Baskaran et al. , 2012, Knyazeva et al. , 2013, Babiloni et al. , 2014, Dong Wen, 2014), especially the relationship between EEG signals from different brain regions. These methods include coherence(Brassen et al., 2004, Hidasi et al. , 2007, Güntekin et al. , 2008, Jelles et al. , 2008, Moretti et al. , 2008, Dauwels et al., 2010b, Bian et al. , 2014), mutual information (Dauwels et al. , 2010b) and likelihood synchronization (Babiloni et al., 2006) as well as the coupling analysis (Rosenblum et al., 2001, Rosenblum et al., 2002). Indeed the coupling analysis between different brain areas has been a focus in a number of studies in both the normal(Mizuhara et al. , 2007, Cantero et al. , 2009, Darvas et al. , 2009) and the diseased brain(Rudrauf et al. , 2006, Uhlhaas et al. , 2006, Amor et al. , 2009, Darvas et al. , 2009).

Although the most of these methods quantified the strength of coupling or coherence, the more recent developments have been trying to estimate the coupling direction of brain rhythms at paired brain sites, these methods include transfer entropy (Schreiber, 2000), conditional mutual information, instantaneous phases of interacting oscillators (Rosenblum et al. , 2001, Rosenblum et al. , 2002), state-space and phase-dynamics , and Granger causality (Lungarella et al. , 2006, Wang et al. , 2008). Transfer entropy (Schreiber, 2000) and conditional mutual information (Palus et al., 2001; Vejmelka and Palus, 2008) could not calculate accurately the coupling direction between neural series (Hlavackova-Schindler et al. 2007). The instantaneous phases (Rosenblum and Pikovsky, 2001; Rosenblum, 2002) were sensitive to noise in the neural series and unsuitable for analyzing noisy and non-stationary EEG signals (Li et al., 2007a,b,c). State-space (Smirnov and Andrzejak 2005) required optimal embedding parameters, but phase-dynamics (Smirnov and Bezruchko, 2003) was only used to describe strong oscillatory behavior and were sensitive to noise in the time series. Granger causality methods can be successfully applied to linear models, and only the directed transfer function of Granger causality in the above 
methods was often used to analyze the functional coupling direction between EEG signals from paired brain sites of cognitive impairment patients (Babiloni et al. , 2008, Babiloni et al. , 2009a, Babiloni et al. , 2009b, Dauwels et al. , 2010b, Liu et al. , 2010, Vecchio et al. , 2011). However, the change in cross-prediction error based on Granger causality could not be directly applied to nonlinear time series .

Recently, the permutation conditional mutual information (PCMI), a nonlinear method, was used to estimate the coupling strength and coupling direction between two time series from mass neuronal model or neuronal populations in CA1 and CA3 in the rat hippocampal tetanus toxin model of focal epilepsy, and between spike trains (Li et al. , 2010, Li et al. , 2011). These studies showed that PCMI method could estimate the coupling direction and was insensitive to noise in the neural series, which was superior to the Granger causality method. Therefore, in this study, we will investigate whether or not the method also can be used to estimate the coupling strength and direction in rsEEG signals between different brain regions of T2DM patients in various frequency bands. In particular, the PCMI was applied for the analysis of the rsEEG recordings from 39 subjects, including 19 amnestic mild cognitive impairment (aMCI) and 20 normal controls (NC) with T2DM, and the difference between the coupling strength values or coupling direction indexes of aMCI and NC with T2DM were calculated. Then, the correlations between the coupling strength or directionality indexes and neuropsychological assessment scores or sex or age of all subjects were also analyzed.

\section{Materials and methods}

\subsection{Subjects}

Participants were comprised of 39 right-handed subjects who satisfied the diagnosis criteria for T2DM (Association, 2013), and they were all voluntary and the mean years were $68.95 \pm 8.95$ years with range from 43 to 84 years. These participants were divided into two groups: aMCI and NC. The aMCI patient group consisted of 19 subjects (12 females and 7 males; mean years of diabetes $9.19 \pm 6.29$ years, range from 1 to 20 years). They were recruited from patients of the Second Artillery General Hospital of PLA in China. The NC group consisted of 20 volunteers (11 females and 9 males; mean years of diabetes $13.60 \pm 8.59$ years, range from 1 to 30 years). They were invited to participate in the experiment from communities near the hospital through the poster. The experiment was conducted in accordance with the Declaration of Helsinki (1964). All participants in our study signed written informed consent forms authorized by the Institutional Review Board of the Second Artillery General Hospital of PLA in China prior to their participation.

\subsection{Diagnostic criteria and neuropsychological measures}

The symptom severity was quantified by the full-scale Chinese version of Mini-mental State Examination (MMSE) (Jia, 2010), in which the cut-off score for absence of dementia was 24 points for high school and above, 20 points for the primary, and 17 for the illiteracy participants, and Montreal Cognitive Assessment (MoCA) scores (Nasreddine et al. , 2005), in which the cut-off score for absence of MCI was 26 points. In addition, other neuropsychological tests scales, which include Auditory Verbal Learning Test (AVLT) (AVLT-Immediate recall, AVLT-Delayed recall, AVLT-Delayed recognition) (Carlesimo et al. , 1996), Wechsler Adult Intelligence Scale Digit Span Test (WAIS-DST) (Orsini et al. , 1987), Boston Naming Test (BNT) (Ferraro et al. , 2010), Trail 
Making Test (Reitan, 1958), Verbal Fluency Test (Novelli et al., 1986), Daily Living Test (Lawton, 1969) were performed to all subjects.

The participants were all T2DM patients including aMCI and NC, whose vision and hearing were normal. They underwent MRI examination to rule out organic brain diseases. The depression that can cause cognitive impairment was excluded using DSM IV criteria for depression (Association, 1994). All patients in the two groups did not have a history of mental illness, systemic disease (such as heart disease, thyroid disease liver and kidney dysfunction) and nervous system disease (such as traumatic brain injury, cerebrovascular disease, epilepsy, hydrocephalus, brain tumors, encephalitis, radiation injury, multiple sclerosis). At the same time, we also considered other medical conditions, including HTN, smoking status and Hb A1c, which could not appear in the subjects.

The aMCI patients with T2DM satisfied the criteria (Petersen et al. , 1997, Petersen, 2004) for the diagnosis of aMCI. Generally, the clinical manifestations are: (1) memory problems, (2) objective memory disorder, (3) absence of other cognitive disorders or repercussions on daily life, (4) normal general cognitive function and (5) absence of dementia (Petersen et al. , 1997, Petersen, 2004).

\subsection{Recording and preprocessing of EEG signals}

\subsubsection{Recording}

The rsEEG signal recording was performed in the Department of Neurology, General Hospital of the Second Artillery Corps of PL in Beijing of China. The participants were invited to wash and brush their hair before the application of the Geodesic Sensor Net (GSN) to their head. During recordings, they were asked to close their eyes and sit in a comfortable armchair, keeping relaxing and awake for 5 minutes in a quiet-dim room, with room temperature keeping at $23 \pm 2^{\circ} \mathrm{C}$.

The rsEEG data recording was performed with a high-density 128-channel EGI system of Net Amps 300 amplifiers (Electrical Geodesics Inc. [EGI], Eugene, OR).The rsEEG was recorded continuously with a 128-channel GSN using the vertex sensor $(\mathrm{Cz})$ as the reference electrode. Direct current acquisition was utilized and the data were sampled at $1000 \mathrm{~Hz}$ during recording. The impedances of all electrodes were kept below $50 \mathrm{k} \Omega$, as recommended for this type of amplifiers by EGI guidelines.

\subsubsection{Preprocessing}

The recorded rsEEG data were analyzed and fragmented off-line using NetStation 4.5 software (Electrical Geodesics). First, a band-pass filter of $1-45 \mathrm{~Hz}$ was applied; then the data were re-referenced to linked mastoids sensors (i.e., electrode 57 [left mastoid process] and electrode 100 [right mastoid process]), and the data were re-sampled to $500 \mathrm{~Hz}$. The ocular, muscular and other types of artifacts were removed by visual inspection of the raw rsEEG data. Finally rsEEG recordings of 3 minutes were segmented for further analysis.

The data was recorded using the 128-channel Geodesic Sensor Net. However, in this study the interested channels came from the 19 electrodes that evenly distributed on the scalp locations from 5 brain regions, which was similar to previous studies (Koenig, et al., 2005, Babiloni, et al., 2010,2014). The 19 electrodes were Fp1, Fp2, F3, Fz, F4 of frontal, F7, T3, T5 of left temporal, C3, C4, P3, Pz, P4 of parietal, F8, T4, T6 of right temporal, 
$\mathrm{O} 1, \mathrm{Oz}, \mathrm{O} 2$ of occipital respectively (Fig. 1). For the aim to estimate the left and right hemispheres paired-electrode coupling, the vertical dashed line (Fig. 1) divided the brain into left and right hemispheres and the frontal region was divided into left frontal and right frontal, the parietal region into left parietal and right parietal, the occipital into left occipital and right occipital.

Any EEG screening and preprocessing did not change the original rsEEG signals including slow and fast wave. Their rsEEG time series had no special findings under visual inspection. We calculated the coupling strength and directionality index in 8 epochs of $6 \mathrm{~s}$ rsEEGs for the following frequency bands in each subject: Delta (1-4 Hz), Theta (4-8 Hz), Alpha $1(8-10.5 \mathrm{~Hz})$, Alpha 2 (10.5-13 Hz), Beta 1 (13-20 Hz), Beta $2(20-30 \mathrm{~Hz})$ and Gamma (30-40 Hz). A moving window technique (window of $6 \mathrm{~s}$ with an overlap of $3 \mathrm{~s}$ ) was implemented. The datasets of coupling strength and directionality index were revealed to obey normal distribution.

\subsection{Permutation conditional mutual information analysis}

In this study, we adopted the permutation conditional mutual information ( $\mathrm{Li}$ et al. , 2010) method to estimator the coupling strength and coupling direction of two rsEEG signals from different electrode pairs on two separate brain regions of aMCI and NC with T2DM. The concrete method was described in detail as follows.

Two series $X$ and $Y$, which were extracted from two different channels in turn and denoted with $X=\left(X_{1}, \ldots, X_{n}\right)^{T}$ and $Y=\left(Y_{1}, \ldots, Y_{n}\right)^{T}$ separately, were embedded in $m$ dimensional space. And the vector $X_{i}=\left(x_{i}, x_{i+\tau}, \ldots, x_{i+(m-1) \tau}\right), i=1, \ldots, n$ and $Y_{j}=\left(y_{j}, y_{j+\tau}, \ldots, y_{j+(m-1) \tau}\right), j=1, \ldots, n$ were obtained, where $m$ denotes embedding dimension and $\tau$ denotes delay time. The elements of vector $X_{i}$ and $Y_{j}$ were re-sorted separately in ascending order. When two values from $X_{i}$ or $Y_{j}$ were equal, we could sort the two values according to the size of the subscript. Therefore, every vector in $m$ dimensional space could all be mapped to $m$ ! kinds of order mode $\pi_{i}, i=1, \ldots, m$ !. In this paper, we assigned 3 to embed dimension $m$, and set the delay time $\tau=11$. In that way, there were 6 kinds of order mode in three dimensional state vector spaces. Firstly, the order mode of the rsEEG signal $X$ from a single channel was analyzed, the same vectors of all order modes were divided into one group, the number of times $C_{1}, C_{2}, \ldots, C_{m !}$ that every order mode appears needed be counted. As a result, the probability $P\left(X=\pi_{1}\right), P\left(X=\pi_{2}\right), \ldots, P\left(X=\pi_{m !}\right)$ of the occurrence of every mode might be calculated as follows:

$$
P\left(X=\pi_{i}\right)=C_{i} /(n-(m-1) \tau), i=1, \ldots, m !
$$

In the same way, the probability of the occurrence of every order mode from rsEEG signal $Y$ could be obtained as follows:

$$
P\left(Y=\pi_{j}\right)=C_{j} /(n-(m-1) \tau), j=1, \ldots, m !
$$

For the sake of brevity, $p\left(x_{i}\right)$ was denoted as the probability distribution of order mode of rsEEG signal 
$X$, and $p\left(y_{j}\right)$ was defined as the probability distribution of order mode of rsEEG signal $Y$. For the rsEEG signal $X, Y$ from two different channels, we could analyze the order mode $\pi_{i}, i=1, \ldots, m !$ and $\pi_{j}, i=1, \ldots, m !$ of vector $X_{k}, k=1, \ldots, n$ and $Y_{k}, k=1, \ldots, n$ at the same time. As a result, there would be $m ! * m$ ! kinds of combination order modes. And then we counted the number of times $C_{i j}$ appearing in each combination order mode. Accordingly, the probability appearing in each combination order mode could be obtained as follows:

$$
P\left(X=\pi_{i}, Y=\pi_{j}\right)=C_{i j} / n-(m-1) \tau, i, j=1, \ldots, m !
$$

Therefore, $p\left(x_{i}, y_{j}\right)$ could be denoted as the joint probability distribution of the permutation mode of rsEEG signal $X$ and $Y$. According to the information theory, the permutation entropy $P E(X)$ and $P E(Y)$, and the permutation joint entropy $P E(X, Y)$ could be obtained as follows:

$$
\begin{gathered}
P E(X)=-\sum_{i=1}^{m !} p\left(x_{i}\right) \log \left(p\left(x_{i}\right)\right) \\
P E(Y)=-\sum_{j=1}^{m !} p\left(y_{j}\right) \log \left(p\left(y_{j}\right)\right) \\
P E(X, Y)=-\sum_{i=1}^{m !} \sum_{j=1}^{m !} p\left(x_{i}, y_{j}\right) \log \left(p\left(x_{i}, y_{j}\right)\right)
\end{gathered}
$$

Then, the permutation conditional entropy of $X$ given $Y$ was given by

$$
P E(X \mid Y)=-\sum_{i=1}^{m !} \sum_{j=1}^{m !} p\left(x_{i}, y_{j}\right) \log \left(p\left(x_{i} \mid y_{j}\right)\right)
$$

The common information contained in $X$ and $Y$ could be estimated by the following permutation mutual information calculation:

$$
P M I(X ; Y)=P E(X)+P E(Y)-P E(X, Y)
$$

The permutation conditional mutual information (PCMI) between two series X and Y might be calculated by the following equations :

$$
P C M I_{X \rightarrow Y}^{\delta}=P C M I\left(X ; Y_{\delta} \mid Y\right)=P E(X \mid Y)+P E\left(Y_{\delta} \mid Y\right)-P E\left(X, Y_{\delta} \mid Y\right)
$$

and

$$
P C M I_{Y \rightarrow X}^{\delta}=P C M I\left(Y ; X_{\delta} \mid X\right)=P E(Y \mid X)+P E\left(X_{\delta} \mid X\right)-P E\left(Y, X_{\delta} \mid X\right)
$$

Where $X_{\delta}\left(Y_{\delta}\right)$ was an observation derived from the state of the process $X(Y) \delta$ steps in the future, i.e. 
$X_{\delta}: x_{t+\delta}=x_{t}\left(Y_{\delta}: y_{t+\delta}=y_{t}\right)$. The value of $\delta$ might be assigned from 3 to 15 . The information that was transferred from the process $X$ (or $Y$ ) to the process $Y$ (or $X$ ) at some later points in time can be defined as

$$
P C M I_{X \rightarrow Y}=\frac{1}{N} \sum_{\delta=1}^{N} P C M I_{X \rightarrow Y}^{\delta}
$$

and

$$
P C M I_{Y \rightarrow X}=\frac{1}{N} \sum_{\delta=1}^{N} P C M I_{Y \rightarrow X}^{\delta}
$$

where the $N$ was the maximal later points. Based on the permutation conditional mutual information, the directionality index between $X$ and $Y$ could be defined by

$$
D_{X Y}=\left(\frac{P C M I_{X \rightarrow Y}-P C M I_{Y \rightarrow X}}{P C M I_{X \rightarrow Y}+P C M I_{Y \rightarrow X}}\right)
$$

The value of $D_{X Y}$ ranged from -1 to $1 . D_{X Y}>0$ meaned that the process $X$ drives $Y ; D_{X Y}<0$ meaned that the process $Y$ drives $X$, and $D_{X Y}=0$ meaned that the interactions between $X$ and $Y$ were symmetrical.

\subsection{Statistical analyses}

An independent sample t-test method was used to calculate the significance of the difference in coupling strength of rsEEG signals between aMCI patients and NC with T2DM, and was also used to compare the age, education, duration of diabetes, MoCA, MMSE and other clinical Neuropsychological assessment scores from the two groups of subjects.

In this study, we adopted the Levene's test as homogeneity of variance test before the t-test, and the hypothesis included null hypothesis that the variances of coupling strength between different brain regions from aMCI and NC came from the same totality. These hypothesis in age, education, duration of diabetes, MoCA, MMSE and other clinical neuropsychological assessment scores were similar to the hypothesis in coupling strength. When the variance was not homogeneous, in this study we adopted $t^{\prime}$ test to correct the error from the result of heteroscedasticity. A chi-square test was utilized to calculate the significance of the difference in directionality index (coupling direction) in rsEEG signals and the difference between the sexes. The study analyzed the correlation between sex and coupling directionality index. As the sample size was less than 50, we corrected the results of chi-square test with Yates correlation method. Also, we adopted the Bonferroni post-correction to correct all testing errors once again.

The Pearson's linear correlation was applied to explore the correlation between coupling strength values and the scores on the symptom severity scales, age and coupling strength in all subjects. The point-biserial correlation was implemented to explore the correlation between the directionality index (coupling direction) values and the scores on the symptom severity scales, age and directionality index, sex and coupling strength in all subjects. The Phi-correlation was used to calculate the relationship between sex and coupling directionality index. 
For the purpose of evaluation by other methods such as LibSVM classifier (Chang et al., 2011) and Area Under the ROC Curve (AUC), we selected optimal single electrode pairs on each frequency band according to the magnitude of $p$ values which reflex the difference in coupling strength between aMCI and NC with T2DM. The selection of optimal group of electrode pairs on each frequency band based on the method: sorted the $p$ values from small to large until $p$ value was less than 0.05 , namely $p_{c}=1-\bigcap_{i=1}^{k}\left(1-p_{i}\right)<0.05$, where $p_{c}$ denoted combined $p$ value and $p_{i}$ denotes the $p$ value of electrode pair, and $k$ denotes the number of $p$ values (Zhang, 2011).

The mean of the coupling strengths of 8 epochs of EEG signals from each subject was calculated, and the means of all subjects were classified with LibSVM classifier. The accuracy, sensitivity, and specificity with LibSVM were calculated, and AUC of the coupling strengths was counted and the ROC curve drew with SPSS. All of the analyses were performed using standard software (SPSS for Windows and Matlab of MathWorks Corporation in USA), $p$ values less than 0.05 were considered as significant.

\section{Results}

\subsection{Demographics and clinical characteristics of the subjects}

Demographics and clinical characteristics of the patients were detailed in detail in Table 1. No difference in demographics was observed between aMCI and NC with T2DM. Sex was compared by using chi-square test, and other items were compared with adopting independent sample $t$ test separately. There were significant differences in scores of MoCA, AVLT-Immediate recall, AVLT-Delayed recall, AVLT-Delayed recognition, Boston Name Test, Verbal Fluency Test, WAIS-DST, and Activity of Daily Living Scale. Although the difference of other assessment scores was not statistically significant, the scores of MMSE were lower, Trail Making Test 1 and Trail Making Test2 were higher in aMCI than in NC.

\subsection{Coupling strength}

\subsubsection{Alphal frequency band}

The difference of coupling strength, and its correlation with MoCA scores in Alpha1 frequency band from fronto-posterior of aMCI and NC with T2DM were presented in Fig. 2. There existed significant difference and correlation in the electrode pairs: $F p 2 \leftrightarrow O 1, F p 1 \leftrightarrow O 1, F 3 \leftrightarrow O 1$, and $F 4 \leftrightarrow O 1$, in which "↔ "denotes that the combination of electrode pairs is bidirectional).

The coupling strength and its correlation with MoCA or AVLT- Delayed recall (ADRL) scores in Alpha1 frequency band from temporal-occipital and temporal-parietal of aMCI and NC with T2DM were showed in Fig. 3. There existed significant difference in coupling strength of EEG signals between aMCI and NC, and correlation between the coupling strength and neuropsychological scales in the electrode pairs: $F 7 \leftrightarrow O 1$, $F 8 \leftrightarrow O 1, C 4 \leftrightarrow T 5$, and $T 6 \leftrightarrow C 4$.

Table 2 showed the mean and standard deviation of coupling strength, correlation coefficient with different neuropsychological assessment scores and their significance from two different brain regions in Alpha1 frequency. The brain regions included right temporal-parietal, parietal-frontal, and right frontal-left temporal. It's worth mentioning that the difference of coupling strength between aMCI and NC with T2DM in $F 8 \rightarrow C 4$ of right 
temporal-parietal was extremely significant.

From the figures 2, 3 and table 2, we found that the minimum of $\mathrm{p}$ value was on $F 8 \rightarrow C 4$ from right temporal-parietal. We also found that the $p_{c}$ was less than 0.05 when combined $F 8 \rightarrow C 4$ from right temporal-parietal, $F p 2 \rightarrow O 1$ and $F p 1 \rightarrow O 1$ from left frontal - left occipital, $T 6 \rightarrow C 4$ from right parietal-right temporal, $F 8 \rightarrow O 1$ from left occipital-right temporal and $F 7 \rightarrow O 1$ from left occipital-left temporal. Table 3 showed the accuracy, sensitivity, specificity of classification and AUC of signal indicator and combination indicator. The figure 4 showed the ROC curve.

\subsubsection{Alpha2 frequency band}

The difference of coupling strength, and its correlation with MoCA scales in Alpha2 frequency band from parietal-right temporal, frontal-parietal and right frontal-left temporal of aMCI and NC with T2DM were displayed in Fig. 5. There existed significant difference and correlation in the electrode pairs: $P z \leftrightarrow F 8, C 4 \leftrightarrow F 8, F z \leftrightarrow P z$ and $F 4 \leftrightarrow T 5$.

Table 4 showed that the mean and standard deviation of coupling strength, correlation coefficient with MoCA scores and their significance from two different brain regions in Alpha2 frequency. The brain regions included right temporal-parietal, occipital-frontal, occipital-right temporal, right temporal-right frontal and left temporal-right occipital. There existed extremely significant differences in coupling strength between aMCI and $\mathrm{NC}$ with $\mathrm{T} 2 \mathrm{DM}$ in $T 6 \rightarrow C 4, T 6 \rightarrow P 4, P z \rightarrow T 4, P 4 \rightarrow F 8$ of right temporal-parietal and $F 8 \rightarrow F 4$ of right temporal- right frontal.

From the figure 5 and table 4 , we found that the minimum of $\mathrm{p}$ value was on $P 4 \rightarrow F 8$ from right temporal-parietal or $P z \rightarrow F 8$ from parietal-right temporal or $P z \rightarrow F z$ from frontal-parietal. Meanwhile, we also found that the $p_{c}$ was less than 0.05 when combined $P 4 \rightarrow F 8, P z \rightarrow T 4, T 6 \rightarrow C 4$ and $T 6 \rightarrow P 4$ from right temporal-parietal, $P z \rightarrow F 8$ from parietal-right temporal, $P z \rightarrow F z$ from frontal-parietal, $F 8 \rightarrow F 4$ from right temporal- right frontal, $C 4 \rightarrow F 8$ and $F 8 \rightarrow C 4$ from right parietal-right temporal. In addition, table 5 showed the accuracy, sensitivity, specificity of classification and AUC of signal indicator and combination indicator, and figure 6 drew the ROC curve.

\subsubsection{Other frequency bands}

Table 6 presented that the mean and standard deviation of coupling strength, correlation coefficient between the strength values and different neuropsychological assessment scores, and their significance from two different brain regions of aMCI and NC on Delta, Theta, Beta1 and Beta2 frequency bands. There existed an extremely significant difference in coupling strength between aMCI and NC with T2DM in $T 3 \rightarrow T 6$ of left temporal-right temporal in the Beta2 frequency band.There was also extremely significant correlation between coupling strength and BNT scales in $T 4 \rightarrow F 7$ of left temporal-right temporal in Delta frequency band, and between coupling direction and ADRN scales in $T 5 \rightarrow T 6$ of left temporal-right temporal in the Beta2 frequency band.

From the table 6 , we found that the coupling strength of EEG signals from $T 4 \rightarrow F 7$ of right temporal-left temporal was unique indicator on Delta frequency band, the coupling strength of EEG signals from $T 6 \rightarrow T 5$ of right temporal-left temporal was the best indicator on Theta frequency band, the coupling strength of EEG signals from $O 1 \rightarrow P 4$ from left occipital-right parietal was the best indicator on Beta1 frequency band, the coupling 
strength of EEG signals from $T 3 \rightarrow T 6$ from left temporal-right temporal was the best indicator on Beta2 frequency band, Meanwhile, we also found that the $p_{c}$ was less than 0.05 when combined $T 3 \rightarrow T 6 、 T 5 \rightarrow T 6$ 、 $F 8 \rightarrow T 3 、 F 7 \rightarrow T 6$ from left temporal-right temporal. In addition, table 7 showed the accuracy, sensitivity, specificity of classification and AUC of signal indicator and combination indicator, and figure 7 drew the ROC curve.

\subsection{Coupling directionality index}

\subsubsection{Alphal frequency band}

Table 8 showed that the number of forward direction and backward direction of coupling directionality (information flow), correlation coefficient between the directionality indexes with different neuropsychological assessment scores, and their significance between rsEEG signals of two electrodes from different brain regions of aMCI and NC in Alpha1 frequency. There existed extremely significant difference in coupling direction between aMCI and NC with T2DM in $F p 1 \rightarrow O 1$ of left frontal-to-left occipital, and was also extremely significant correlation between MoCA scales and coupling directionality indexes in $F p 2 \rightarrow T 3$ and $F z \rightarrow T 3$ of frontal-to-left temporal, and between Verbal Fluency Test scores and coupling directionality indexes in $F p 2 \rightarrow F p 1$ of right frontal-to-left frontal.

From table 8, we found that the coupling directionality index of EEG signals from $F p 1 \rightarrow O 1$ of left frontal-to-left occipital was the best indicator on Alpha1 frequency band. We also found that combined $p$ value $\left(p_{c}\right)$ in coupling strength between $F p 1 \rightarrow O 1$ (from left frontal-to-left occipital), $O 1 \rightarrow P 4$ (from left occipital -toright parietal) and $\mathrm{Fz} \rightarrow T 3$ (from frontal-to-left temporal) or $C 3 \rightarrow T 4$ (from left parietal-to- right temporal) meets the criteria of $p$ value less than 0.05 .

\subsubsection{Other frequency bands}

Table 9 presented that the forward direction and backward direction between two electrodes from two brain regions, correlation coefficient with different neuropsychological assessment scores, and their significance in Delta, Alpha2, Beta1 and Gamma frequency of aMCI and NC. There existed an extremely significant difference in coupling direction between aMCI and NC with T2DM in $F 7 \rightarrow F 8$ of left temporal-to-right temporal in the Delta frequency band and $F 3 \rightarrow C 4$ of frontal-to-parietal in the Gamma frequency band. There was also extremely significant correlation between coupling direction and MoCA scales in $C 3 \rightarrow T 5$ of parietal-to-left temporal in Alpha2 frequency band, and between coupling direction and Boston Name Test scales in $F 3 \rightarrow C 4$ of left frontal-to-right parietal in the Gamma frequency band.

From table 9, we found that the coupling directionality index of EEG signals from $F 7 \rightarrow F 8$ of left temporal-to- right temporal was the best indicator on Delta frequency band, the coupling directionality index of EEG signals from $T 3 \rightarrow F 8$ of left temporal-to- right temporal was the unique indicator on Beta1 frequency band, the coupling directionality index of EEG signals from $F 3 \rightarrow C 4$ of left frontal-to- right parietal was the unique indicator on Gamma frequency band, the coupling directionality index of EEG signals from $C 4 \rightarrow T 4$ of parietal-to-right temporal was the best indicator on Alpha2 frequency band. Meanwhile, we also found that the $p_{c}$ was less than 0.05 when $C 4 \rightarrow T 4$ from parietal-to-right temporal and $C 3 \rightarrow T 5$ from parietal-to-left temporal were combined. 


\subsection{The respective correlation between the sex or age and coupling strength or coupling directionality index}

\subsubsection{The relationship between coupling strength and sex, age}

Table 10 showed the correlation coefficients and their significance between sex and coupling strength, age and coupling strength of EEG signals from two electrodes on two different brain regions of all subjects in Delta, Alpha1, Alpha2, Beta1, Beta2,and Gamma frequency. There was an extremely significant correlation between coupling strength and sex in $P z \rightarrow T 6$ of parietal-right temporal in the Alpha1 frequency band and $O 2 \rightarrow T 6$ of right occipital-right temporal in the Alpha2 frequency band. There was also extremely significant correlation between sex and coupling strength in $O 2 \rightarrow C 3$ and $O 2 \rightarrow P 3$ of right occipital-left parietal in the Gamma frequency band.

\subsubsection{The relationship between coupling directionality index and sex}

The results of correlation calculation between coupling directionality index and sex displayed that there existed a significant correlation between the sex of all subjects and coupling directionality index of $C 4 \rightarrow T 4$ from right parietal and right temporal on Alpha2 frequency band. The value of chi-square used in calculating the correlation is 5.17 and greater than the threshold 3.84 on a 0.05 significance level. Table 11 showed the direction between the right parietal and right temporal of subjects of different sex on Alpha2 frequency band, in which the main coupling direction of man was $T 4 \rightarrow C 4$, and the main coupling direction of woman was $C 4 \rightarrow T 4$.

\section{Discussions}

\subsection{The performance of PCMI method}

Transfer entropy (Schreiber, 2000), conditional mutual information, instantaneous phases of interacting oscillators (Rosenblum et al. , 2001, Rosenblum et al. , 2002), state-space and phase-dynamics , and Granger causality (Lungarella et al. , 2006, Wang et al. , 2008) have been developed to analyze the strength and direction of coupling of EEG signals between different brain sites. In these methods, only Granger causality was used to analyze the direction of the information flow within the functional coupling of brain rhythms at paired brain sites of cognitive impairment patients (Babiloni et al. , 2008, Babiloni et al. , 2009a, Babiloni et al. , 2009b, Dauwels et al. , 2010b, Liu et al. , 2010, Vecchio et al. , 2011). At present, we proposed the PCMI to estimate the coupling strength and coupling direction between two time series from mass neuronal model, real neuronal populations and spike trains ( $\mathrm{Li}$ et al. , 2010, Li et al. , 2011). These studies suggested that PCMI was superior to the Granger causality method for identifying the coupling direction between unidirectional or bidirectional neuronal populations.

To our knowledge, we are the first to apply the PCMI method to analyze the rsEEG signals from different brain regions of aMCI with T2DM, and to explore coupling direction of aMCI with T2DM. In this study, we found that the differences of coupling strength and direction in rsEEG signals from pairs of the different brain regions were significant, and that the correlation between the coupling strength or direction values and neuropsychological assessment scores also were significant. In addition, we found that the correlation between 
sex and coupling strength, age and coupling strength of all subjects were also significant. There existed significant correlation between sex and coupling directionality index of right parietal-to-right temporal on Alpha2 frequency band. These results suggested that PCMI method was likely to explain demographics and clinical characteristic indirectly, including the distribution of age, sex and many neuropsychological scales. Therefore, it preliminary illustrated the rationality of PCMI used to distinguish aMCI patients and NC with T2DM.

\subsection{The difference and correlation of coupling strength}

There existed significant difference in the coupling strength of EEG signals from different brain regions between aMCI and NC with T2DM and the coupling strength were significantly correlated with the scores of neuropsychological scales.

\subsubsection{Alpha1 and Alpha2 frequency bands}

In this paper, we found the abnormity of coupling strength in Alpha1 and Alpha2 frequency bands in the aMCI patients with T2DM relative to NC with T2DM. The differences of coupling strength of frontal-occipital and temporal-occipital between the aMCI and NC with T2DM were significant in Alpha1 frequency band. The results were the same as the observation that the coherence strength in Alpha band between frontal and occipital, temporal and occipital, which were all lower in the aMCI subjects than in the NC with T2DM (Bian et al. , 2014). There existed significant difference in coupling strength of EEG signals from temporal-parietal, right temporal-to-parietal and right frontal-to-left temporal between the aMCI and NC with T2DM in Alpha1 frequency band. There existed significant difference in coupling strength of EEG signals from bidirectional right temporal and parietal brain regions between the aMCI and $\mathrm{NC}$ with T2DM in Alpha2 frequency band. The information flow of right temporal-to-parietal showed the significant difference of coupling strength between the aMCI and NC with T2DM in Alpha2 frequency band. There existed significant difference in bidirectional coupling strength of EEG signals from frontal-left temporal, right temporal-right frontal, and left temporal-right occipital between the aMCI and NC with T2DM in the Alpha2 frequency band.These results were the same as the observation that the T2DM patient had an improvement of cognitive ability together with increased connectivity in the Alpha band over central and lateral regions after received intensified treatment (Cooray et al. , 2011). There existed significant difference in coupling strength of EEG signals from frontal-to-parietal between the aMCI and $\mathrm{NC}$ with T2DM in Alpha2 frequency band. The result was the same as the observations that a decrease of intrahemispheric coherence in fronto-parietal regions encompassing all frequency bands(Moretti et al. , 2008), and fronto-parietal functional coupling of EEG rhythms was higher in magnitude in the $\mathrm{NC}$ than in the $\mathrm{MCI}$ subjects(Babiloni et al. , 2008).

\subsubsection{Other frequency bands}

In Delta frequency band, the information flow of right temporal-to-left temporal, displayed the significant difference in coupling strength between aMCI and NC with T2DM. The result was the same as the observation that the coherence strength in Delta band between right temporal and left temporal were higher in the aMCI subjects than in the NC with T2DM (Bian et al. , 2014), and there existed a increase of intrahemispheric coherence on temporal regions encompassing Delta frequency band in MCI subjects relative to normal 
control(Moretti et al. , 2008).

In Theta frequency band, the information flow of right temporal-to-left temporal, displayed the significant difference of coupling strength between the aMCI and NC with T2DM. The result was the same as the observation that there existed an increase of intrahemispheric coherence on temporal regions encompassing the Theta frequency band in MCI subjects relative to normal control(Moretti et al. , 2008).

In Beta1 frequency band, the information flow of right temporal-to-left temporal and left occipital-to-right parietal, displayed the significant difference of coupling strength between the aMCI and NC with T2DM. In Beta2 frequency band, the information flow of left temporal-right temporal and right frontal-left frontal, all showed the significant difference of coupling strength between the aMCI and NC with T2DM. These results were similar to the findings that the T2DM patient had an improvement of cognitive ability together with increased connectivity in the Beta band over central regions of the scalp after received intensified treatment (Cooray et al. , 2011).

Above results were obtained using independent sample t-test method, the coupling strength classified by SVM classifier could provide more accurate marker. The results of t-test and SVM were similar, which suggested that the coupling strength based on PCMI method could distinguish aMCI and NC with T2DM.

Several studies indicated that the meaning of classification was more obvious and the performance of diagnosis was better when the AUC is more than 0.7 (Matthias, et al.,2000, Laura, et al., 2011). In addition, the accuracy or sensitivity or specificity of classification was improved when several effective electrode pairs were combined. Therefore, above tables and figures showed that the PCMI method had certain accuracy of classification by using SVM classifier, which was certified by the lesser $p$ value. However, the result of classification may be better if use better classifier. Therefore, the result of $\mathrm{p}$ value and classification performance suggested that the method of feature extraction based on PCMI had better effectiveness, and the method has higher performance of resolution to EEG signal of aMCI in T2DM.

\subsection{The difference and correlation of coupling direction}

We also found that there existed significant difference between the coupling direction of EEG signals from different brain regions of aMCI and $\mathrm{NC}$, and these coupling directionality indexes were significantly correlated with the scores of neuropsychological scale.

A few studies did consider the concrete coupling direction or information flow in cognitive information processing (Babiloni et al. , 2008, Babiloni et al. , 2009a, Babiloni et al., 2009b, Dauwels et al. , 2010b, Liu et al. , 2010, Vecchio et al. , 2011). The studies suggested that the abnormity of connectivity or coherence or coupling involved the abnormal coupling direction besides abnormal coupling strength. This study explored the rsEEG coupling direction between different brain regions of aMCI with T2DM for the first time. In this paper, we found that the abnormity of coupling direction in Alpha1,Alpha2, Beta1, Delta and Gamma frequency bands appeared in the aMCI patients with T2DM relative to NC with T2DM.

In the Alpha1 frequency band, there existed significant difference in coupling direction of EEG signals from frontal-to-left temporal, frontal (right)- to-frontal(left), occipital (left)-to-parietal (right) and parietal(left)-to-right temporal between the aMCI and NC with T2DM. In the Alpha2 frequency band, there existed significant difference in coupling direction of EEG signals from parietal-to-left temporal, parietal-to-right temporal between 
the aMCI and NC with T2DM. These results were the same as the observation that the T2DM patient had an improvement of cognitive ability together with increased connectivity in the Alpha band over central and lateral regions after received intensified treatment (Cooray et al. , 2011). It showed a significant difference in coupling direction of EEG signals from frontal-to-occipital between the aMCI and NC with T2DM in the Alpha1 frequency band. The result was similar to the find that the coherence strength in Alpha band between frontal and occipital was lower in the aMCI subjects than in the NC with T2DM (Bian et al. , 2014).

In Beta1 frequency band, it presented significant difference in coupling direction of EEG signals from left temporal-to-right temporal between the aMCI and NC with T2DM. The result was similar to the findings that the T2DM patient had an improvement of cognitive ability together with increased connectivity in the Beta band over central regions of the scalp after received intensified treatment (Cooray et al. , 2011).

In Delta frequency band, it displayed a significant difference in coupling direction of EEG signals from left temporal-to-right temporal between the aMCI and NC with T2DM. The result was the same as the observation that the coherence strength in Delta band between right temporal and left temporal were higher in the aMCI subjects than in the NC with T2DM (Bian et al. , 2014), and there existed a increase of intrahemispheric coherence on temporal regions encompassing Delta frequency bands in MCI subjects relative to normal control (Moretti, et al., 2008).

In Gamma frequency band, there existed a significant difference in coupling direction of EEG signals from frontal-to- parietal between the aMCI and $\mathrm{NC}$ with $\mathrm{T} 2 \mathrm{DM}$. The consequence was the same as the observation that there existed a decrease of intrahemispheric coherence in fronto-parietal regions encompassing all frequency bands(Moretti et al. , 2008).

Based on the analysis of selected electrode pairs in different brain regions, we could get more accurate indicators on coupling direction in these brain regions. The indicators on coupling direction based on PCMI may act as more important roles differing aMCI and $\mathrm{NC}$ with $\mathrm{T} 2 \mathrm{DM}$ in different frequency bands. If an active alpha generator was present in inferior temporal regions (i.e., near the mastoid reference electrodes), the result of linkage measures was uncorrelated with this study. Because this study measure mainly the coupling linkage between two brain regions far away from mastoid reference electrodes (see Fig. 1).

\section{Conclusions}

In this study, the PCMI method was utilized to quantify a coupling strength and coupling direction between two rsEEG series from two different brain regions of aMCI and NC with T2DM. Based on the analysis of the rsEEG time series from all subjects, the PCMI method can be used effectively in estimating the coupling strength and direction of different brain regions of aMCI and NC with T2DM, and could be considered as a potential biomarker for diagnosing aMCI with T2DM. The AUC of ROC curve based on coupling strength also proved the rationality of independent sample t-test. Thus, the coupling strength or coupling direction of EEG signals may be used as the important indexes for the distinguishing aMCI and NC with T2DM. 


\section{Conflict of Interest}

None of the authors have potential conflicts of interest to be disclosed.

\section{Acknowledgments}

This research was funded in part by National Science Foundation of China $(61273063$, 81230023, 31070938, 61403331), Open project from State Laboratory of Cognitive Neuroscience and Learning, Beijing Normal University of China (2012-2014), and Doctorial Foundation of Yanshan University in China (B900), Science and Technology Support Programme of Hebei province in China (13212003). The authors thank these colleagues from the State Key Laboratory of Cognitive Neuroscience and Learning \& IDG/McGovern Institute for Brain Research of Beijing Normal University in China. 


\section{References}

Amor F, Baillet S, Navarro V, Adam C, Martinerie J, Le Van Quyen M. Cortical local and long-range synchronization interplay in human absence seizure initiation. Neuroimage. 2009;45:950-62.

Association AD. Diagnosis and classification of diabetes mellitus. Diabetes care. 2013;36:S67-S74.

Association AP. Diagnostic and Statistical Manual of Mental Disorders, DSM-IV Washington, DC: American Psychiatric Association. 1994.

Babiloni C, Del Percio C, Lizio R, Marzano N, Infarinato F, Soricelli A, et al. Cortical sources of resting state electroencephalographic alpha rhythms deteriorate across time in subjects with amnesic mild cognitive impairment. Neurobiol Aging. 2014;35:130-42.

Babiloni C, Ferri R, Binetti G, Cassarino A, Forno GD, Ercolani M, et al. Fronto-parietal coupling of brain rhythms in mild cognitive impairment: a multicentric EEG study. Brain Res Bull. 2006;69:63-73.

Babiloni C, Ferri R, Binetti G, Vecchio F, Frisoni GB, Lanuzza B, et al. Directionality of EEG synchronization in Alzheimer's disease subjects. Neurobiol Aging. 2009a;30:93-102.

Babiloni C, Frisoni GB, Pievani M, Vecchio F, Infarinato F, Geroldi C, et al. White matter vascular lesions are related to parietal-to-frontal coupling of EEG rhythms in mild cognitive impairment. Hum Brain Mapp. 2008;29:1355-67.

Babiloni C, Pievani M, Vecchio F, Geroldi C, Eusebi F, Fracassi C, et al. White - matter lesions along the cholinergic tracts are related to cortical sources of EEG rhythms in amnesic mild cognitive impairment. Hum Brain Mapp. 2009b;30:1431-43.

Babiloni C, Visser PJ, Frisoni G, De Deyn PP, Bresciani L, Jelic V, et al., Cortical sources of resting EEG rhythms in mild cognitive impairment and subjective memory complaint. Neurobiol Aging. 2010;31:1787-98.

Baskaran A, Milev R, McIntyre RS. A review of electroencephalographic changes in diabetes mellitus in relation to major depressive disorder. Neuropsych. Dis Treat. 2012;9:143-50.

Bian Z, Li Q, Wang L, Lu C, Yin S, Li X. Relative power and coherence of EEG series are related to amnestic mild cognitive impairment in diabetes. Front Aging Neurosci. 2014;6:11.

Brassen S, Braus DF, Weber-Fahr W, Tost H, Moritz S, Adler G. Late-onset depression with mild cognitive deficits: electrophysiological evidences for a preclinical dementia syndrome. Dement Geriatr Cogn. 2004;18:271-7.

Busse, A, Hensel, A, Guhne, U, Angermeyer, M. C, Riedel-Heller, S. G. Mild cognitive impairment: long-term course of four clinical subtypes. Neurology.2006;67:2176-85.

Chang C, Lin C. LIBSVM: a library for support vector machines. ACM T. Intel Syst Tec. 2011;2:27

Cantero JL, Atienza M, Cruz-Vadell A, Suarez-Gonzalez A, Gil-Neciga E. Increased synchronization and decreased neural complexity underlie thalamocortical oscillatory dynamics in mild cognitive impairment. Neuroimage. 2009;46:938-48.

Carlesimo G, Caltagirone C, Gainotti G, Fadda L, Gallassi R, Lorusso S, et al. The Mental Deterioration Battery: normative data, diagnostic reliability and qualitative analyses of cognitive impairment. Eur Neurol. 1996;36:378-84.

Cooray G, Nilsson E, Wahlin Å, Laukka EJ, Brismar K, Brismar T. Effects of intensified metabolic control on CNS function in type 2 diabetes. Psychoneuroendocrino. 2011;36:77-86.

Darvas F, Ojemann JG, Sorensen LB. Bi-phase locking - a tool for probing non-linear interaction in the human brain. NeuroImage. 2009;46:123-32.

Dauwels J, Vialatte F, Cichocki A. Diagnosis of alzheimers disease from EEG signals: Where are we standing? Curr Alzheimer Res. 2010a; 7:487-505.

Dauwels J, Vialatte F, Musha T, Cichocki A. A comparative study of synchrony measures for the early diagnosis of Alzheimer's disease based on EEG. NeuroImage. 2010b;49:668-93.

Dong Wen, Qin Xue, Chengbiao Lu, Xinyong Guan, Yuping Wang, Xiaoli Li. A global coupling index of multivariate neural series with application to the evaluation of mild cognitive impairment. Neural Networks. 2014;56:1-9.

Ferraro FR, Lowell K. Boston Naming Test. Corsini Encyclopedia of Psychology. 2010. 
Fischer P, Jungwirth S, Zehetmayer S, Weissgram S, Hoenigschnabl S, Gelpi E, Krampla W, Tragl KH. Conversion from subtypes of mild cognitive impairment to Alzheimer dementia. Neurology.2007;68: 288-91.

Ganguli, M., Dodge, H. H, Shen, C, Dekosky, S. T. Mild cognitive impairment, amnestic type: an epidemiologic study. Neurology, 2004;63:115-121.

Güntekin B, Saatçi E, Yener G. Decrease of evoked delta, theta and alpha coherences in Alzheimer patients during a visual oddball paradigm. Brain Res. 2008;1235:109-16.

Gerald Cooray EN, Ake Wahlin, Kerstin Brismar, Tom Brismar. Effect of intensified metabolic control on cognitive performance and EEG in patients with type 2 diabetes. 13th European Congress of Clinical Neurophysiology. 2008;119:s1-131.

Gispen WH, Biessels G-J. Cognition and synaptic plasticity in diabetes mellitus. Trends Neurosci. 2000;23:542-9.

Hidasi Z, Czigler B, Salacz P, Csibri É, Molnár M. Changes of EEG spectra and coherence following performance in a cognitive task in Alzheimer's disease. Int J Psychophysiol. 2007;65:252-60.

Huerta KC, Téllez GY, Salinas CAA, Díaz JMM. Cognitive Function in Type 2 Diabetes: A Review. Salud Mental. 2013;36:149-57.

Hlavackova-Schindler, K, Palus, M, Vejmelka, M, Bhattacharya, J. Causality detection based on information- theoretic approaches in time series analysis. Phys Rep. 2007;441:1-46.

Jelles B, Scheltens P, Van der Flier W, Jonkman E, da Silva F, Stam C. Global dynamical analysis of the EEG in Alzheimer's disease: frequency-specific changes of functional interactions. Clin Neurophysiol. 2008;119:837-41.

Jia J. Chinese Dementia and Cognitive Disorders Treatment Guidelines Beijing: People's Medical Publishing House. 2010 .

Knyazeva MG, Carmeli C, Khadivi A, Ghika J, Meuli R, Frackowiak RS. Evolution of source EEG synchronization in early Alzheimer's disease. Neurobiol Aging. 2013;34:694-705.

Koenig T, Prichep L, Dierks T, Hubl D, Wahlund LO, John ER, Jelic V. Decreased EEG synchronization in Alzheimer's disease and mild cognitive impairment.Neurobiol Aging.2005;26:165-71

Kumar V, Nelson, F., Abbas, A. K., Cotran, R. S., and Robbins, S. L. Robbins and Cotran Pathologic Basis of Disease, 7th Edition. Philadelphia, PA: Saunders. 2005.

Laura J, Subashan P, Stephanie A S. Is Timed Up and Go Better Than Gait Speed in Predicting Health, Function, and Falls in Older Adults? J Am Geriatr Soc. 2011;59:887-92

Lawton MP, and Brody, E. M. Assessment of older people: self-maintaining and instrumental activities of daily living. Gerontologist. 1969;9:179-86.

Li X, Ouyang G. Estimating coupling direction between neuronal populations with permutation conditional mutual information. NeuroImage. 2010;52:497-507.

Li, X, Ouyang, G, Richards, D.A. Predictability analysis of absence seizures with permutation entropy. Epilepsy Res. 2007a;77:70-4.

Li, X, Yao, X, Fox, J, Jefferys, J.G. Interaction dynamics of neuronal oscillations analysed using wavelet transforms. J Neurosci Meth. 2007b;160:178-85.

Li, Y, Li, X, Ouyang, G, Guan, X. 2007. Information flow among neural networks with Baysine estimation. Chinese Sci Bull. 2007c;50:2006-11.

Li Z, Ouyang G, Li D, Li X. Characterization of the causality between spike trains with permutation conditional mutual information. Phys Rev E. 2011;84:021929.

Liu J-P, Zhang C, Zheng C-X. Estimation of the cortical functional connectivity by directed transfer function during mental fatigue. Appl Ergon. 2010;42:114-21.

Luchsinger JA, Reitz C, Patel B, Tang M-X, Manly JJ, Mayeux R. Relation of diabetes to mild cognitive impairment. Arch Neurol. 2007;64:570-5.

Lungarella M, Sporns O. Mapping information flow in sensorimotor networks. PLoS Comput. Biol. 2006;2:e144.

Matthias G, D Pfeiffer, R D Smith. Principles and practical application of the receiver-operating characteristic analysis 
for diagnostic tests. Prev Vet Med.2000;45:23-41.

Meihua Qu CF, Xiurong Zhang, Chunzhen Zhao, Shumei Mao, \& Zhiqin Gao. Type 2 Diabetes and Mild Cognitive Impairment. Prog Biochem Biophys. 2012;39:791-5.

Mizuhara H, Yamaguchi Y. Human cortical circuits for central executive function emerge by theta phase synchronization. Neuroimage. 2007;36:232-44.

Moran C, Phan TG, Chen J, Blizzard L, Beare R, Venn A, et al. Brain Atrophy in Type 2 Diabetes Regional distribution and influence on cognition. Diabetes care. 2013;36:4036-42.

Moretti DV, Frisoni GB, Pievani M, Rosini S, Geroldi C, Binetti G, et al. Cerebrovascular disease and hippocampal atrophy are differently linked to functional coupling of brain areas: an EEG coherence study in MCI subjects. J Alzheimer's Dis. 2008;14:285-99.

Nasreddine ZS, Phillips NA, Bédirian V, Charbonneau S, Whitehead V, Collin I, et al. The Montreal Cognitive Assessment, MoCA: a brief screening tool for mild cognitive impairment. J Am Geriatr Soc. 2005;53:695-9.

Novelli G, Papagno C, Capitani E, Laiacona M, Vallar G, Cappa S. Three clinical tests for the assessment of lexical retrieval and production. Norms from 320 normal subjects. Arch. Psicol. Neurol Psichiatr. 1986;47:477-506.

Orsini A, Grossi D, Capitani E, Laiacona M, Papagno C, Vallar G. Verbal and spatial immediate memory span: normative data from 1355 adults and 1112 children. Ital J Neurol Sci. 1987;8:537-48.

Paluš M, Komárek V, Hrnčíř Z, Štěrbová K. Synchronization as adjustment of information rates: detection from bivariate time series. Phys Rev E. 2001;63:046211.

Paluš M, Vejmelka M. Directionality of coupling from bivariate time series: How to avoid false causalities and missed connections. Phys Rev E. 2007;75:056211.

Peila R, Rodriguez BL, Launer LJ. Type 2 diabetes, APOE gene, and the risk for dementia and related pathologies: the Honolulu-Asia Aging Study. Diabetes. 2002;51:1256-62.

Petersen RC. Mild cognitive impairment as a diagnostic entity. J Intern Med. 2004;256:183-94.

Petersen RC, Smith GE, Waring SC, Ivnik RJ, Kokmen E, Tangelos EG. Aging, memory, and mild cognitive impairment. Int Psychogeriatr. 1997;9:65-9.

Reitan RM. Validity of the Trail Making Test as an indicator of organic brain damage. Percept Mot Skills. 1958;8:271-6.

Roberts RO, Knopman DS, Geda YE, Cha RH, Pankratz VS, Baertlein L, et al. Association of diabetes with amnestic and nonamnestic mild cognitive impairment. Alzheimer's Dement. 2014;10:18-26.

Rosenblum MG, Cimponeriu L, Bezerianos A, Patzak A, Mrowka R. Identification of coupling direction: Application to cardiorespiratory interaction. Phys Rev E. 2002;65:041909.

Rosenblum MG, Pikovsky AS. Detecting direction of coupling in interacting oscillators. Phys Rev E. 2001;64:045202 (R).

Rossini PM, Rossi S, Babiloni C, Polich J. Clinical neurophysiology of aging brain: from normal aging to neurodegeneration. Prog neurobiol. 2007;83:375-400.

Rudrauf D, Douiri A, Kovach C, Lachaux J-P, Cosmelli D, Chavez M, et al. Frequency flows and the time-frequency dynamics of multivariate phase synchronization in brain signals. Neuroimage. 2006;31:209-27.

Schmidt MT, Kanda PA, Basile LF, da Silva Lopes HF, Baratho R, Demario JL, et al. Index of alpha/theta ratio of the electroencephalogram: a new marker for Alzheimer's disease. Front Aging Neurosci. 2013;5:60.

Schreiber T. Measuring information transfer. Phys Rev Lett. 2000;85:461.

Shimada H, Miki T, Tamura A, Ataka S, Emoto M, Nishizawa Y. Neuropsychological status of elderly patients with diabetes mellitus. Diabetes Res Clin Pr. 2010;87:224-7.

Shoback ebDGG, Dolores. Greenspan's basic \& clinical endocrinology (9th ed.). New York: McGraw-Hill Medical. 2011.

Smirnov DA, Andrzejak RG. Detection of weak directional coupling: Phase-dynamics approach versus state-space approach. Phys Rev E. 2005;71:036207. 
Smirnov DA, Bezruchko BP. Estimation of interaction strength and direction from short and noisy time series. Phys Rev E. 2003;68:046209.

Steriade M. Grouping of brain rhythms in corticothalamic systems. Neuroscience. 2006;137:1087-106.

Strachan MW, Reynolds RM, Marioni RE, Price JF. Cognitive function, dementia and type 2 diabetes mellitus in the elderly. Nat Rev Endocrinol. 2011;7:108-14.

Toro P, Schönknecht P, Schröder J. Type II diabetes in mild cognitive impairment and Alzheimer's disease: results from a prospective population-based study in Germany. J Alzheimer's Dis. 2009;16:687-91.

Tuma, I. Diabetes mellitus and dementia. Vnitr Lek. 2012;58: 305-308.

Uhlhaas PJ, Singer W. Neural synchrony in brain disorders: relevance for cognitive dysfunctions and pathophysiology. Neuron. 2006;52:155-68.

Vecchio F, Babiloni C. Direction of Information Flow in Alzheimer's Disease and MCI Patients. Int J Alzheimer's Dis. 2011;2011:214580.

Vejmelka M, Paluš M. Inferring the directionality of coupling with conditional mutual information. Phys Rev E. 2008;77:026214.

Wang X, Chen Y, Ding M. Estimating Granger causality after stimulus onset: a cautionary note. NeuroImage. 2008;41:767-76.

Zhang X R. Statistical Analysis with SAS. Beijing: Press of Tsinghua University. 2011:121-122

Xu, W., Caracciolo, B., Wang, H. X., Winblad, B., Backman, L., Qiu, C., et al. Accelerated progression from mild cognitive impairment to dementia in people with diabetes. Diabetes.2010;59: 2928-35.

Yaffe, K., Petersen, R. C., Lindquist, K., Kramer, J., and Miller, B. Subtype of mild cognitive impairment and progression to dementia and death. Dement Geriatr Cogn Disord. 2006;22:312-19.

Zhang J, Wang Y, Wang J, Zhou X, Shu N,Wang Y, Zhang Z.White matter integrity disruptions associated with cognitive impairments in type 2 diabetes patients. Diabetes. 2014;63:3596-605. 


\section{Figure Legends}

Fig. 1. The electrodes distribution of 128-channel Geodesic Sensor Net interested electrodes' partition. A showed that the 19 interested electrodes distribute inside the intimal black dotted line. Thick solid lines divided the interested electrodes into 5 regions: frontal, left temporal, parietal, right temporal and occipital regions, respectively. Vertical dotted line divided the brain into left and right hemispheres, left frontal and right frontal, left parietal and right parietal, and left occipital and right occipital. B showed the 21 electrodes distribute including of 19 interested electrodes and 2 mastoid reference electrodes.

Fig. 2. The coupling strength and its correlation with MoCA scores in Alpha1 frequency band between different brain sites from patients of aMCI and NC with T2DM. A1, B1, C1, D1: the brain map of the coupling strength, dotted line with arrows indicate that there existed bi-directional coupling between the brain sites: $F p 2 \leftrightarrow O 1, F p 1 \leftrightarrow O 1, F 3 \leftrightarrow O 1$, and $F 4 \leftrightarrow O 1 . \mathrm{A} 2, \mathrm{~B} 2, \mathrm{C} 2$, D2: the mean value of coupling strength between the brain sites: $F p 2 \leftrightarrow O 1, F p 1 \rightarrow O 1, F 3 \leftrightarrow O 1$, and $F 4 \leftrightarrow O 1$. A3, B3, C3, D3: the correlation between coupling strength and MoCA scores for the following electrode pairs: $F p 2 \rightarrow O 1, F p 1 \rightarrow O 1$, $F 3 \rightarrow O 1$, and $F 4 \rightarrow O 1$, in which " $\rightarrow$ "denote that the combination of electrode pairs is unidirectional).. A4,B4,C4,D4: the correlation between coupling strength and MoCA scores for the following electrode pairs: $O 1 \rightarrow F p 2, O 1 \rightarrow F p 1, O 1 \rightarrow F 3$, and $O 1 \rightarrow F 4$.Red: aMCI, Green: NC. ${ }^{* *} p<0.01,0.01<{ }^{*} p<0.05$.

Fig. 3. The coupling strength and its correlation with MoCA or ADRL scores in Alpha1 frequency band between different brain sites from of aMCI and NC with T2DM. A1,B1,C1,D1: the brain map of the coupling strength with dotted line, in which there existed bi-directional coupling between electrode $F 7 \leftrightarrow O 1, F 8 \leftrightarrow O 1, C 4 \leftrightarrow T 5$, and $T 6 \leftrightarrow C 4$. A2,B2,C2,D2: the mean value, standard deviation and significance of difference of bi-directional electrode pairs $F 7 \leftrightarrow O 1, F 8 \leftrightarrow O 1, C 4 \leftrightarrow T 5$, and $T 6 \leftrightarrow C 4$.A3,B3,C3,D3: the correlation coefficient and significance of directional electrode pairs $F 7 \rightarrow O 1, F 8 \rightarrow O 1$, $C 4 \rightarrow T 5$, and $T 6 \rightarrow C 4$.A4,B4,C4,D4: the correlation coefficient and significance of directional electrode pairs $O 1 \rightarrow F 7$, $O 1 \rightarrow F 8, T 5 \rightarrow C 4$, and $C 4 \rightarrow T 6$. Red: aMCI, Green: NC. ${ }^{* *} p<0.01,0.01<{ }^{*} p<0.05$.

Fig. 4. ROC curve from different electrode pairs on Alpha1 frequency band.

Fig. 5. The coupling strength and its correlation with MoCA scores in Alpha2 frequency band between different brain sites from of aMCI and NC with T2DM. A1,B1,C1,D1: the brain map of the coupling strength with dotted line, in which there existed bi-directional coupling between electrode $P z \leftrightarrow F 8, C 4 \leftrightarrow F 8, F z \leftrightarrow P z$, and $F 4 \leftrightarrow T 5$. A2,B2,C2,D2: the mean value, standard deviation and significance of difference of bi-directional electrode pairs $P z \leftrightarrow F 8, C 4 \leftrightarrow F 8, F z \leftrightarrow P z$, and $F 4 \leftrightarrow T 5$. A3,B3,C3,D3: the correlation coefficient and significance of directional electrode pairs $P z \rightarrow F 8, C 4 \rightarrow F 8$, $F z \rightarrow P z$, and $F 4 \rightarrow T 5$. A4,B4,C4,D4: the correlation coefficient and significance of directional electrode pairs $F 8 \rightarrow P z, F 8 \rightarrow C 4, P z \rightarrow F z$, and $T 5 \rightarrow F 4$.Red: aMCI, Green: NC. ${ }^{* *} p<0.01,0.01<{ }^{*} p<0.05$.

Fig. 6. ROC curve from different electrode pairs on Alpha2 frequency band.

Fig. 7. ROC curve from different electrode pairs on multiple frequency bands. 


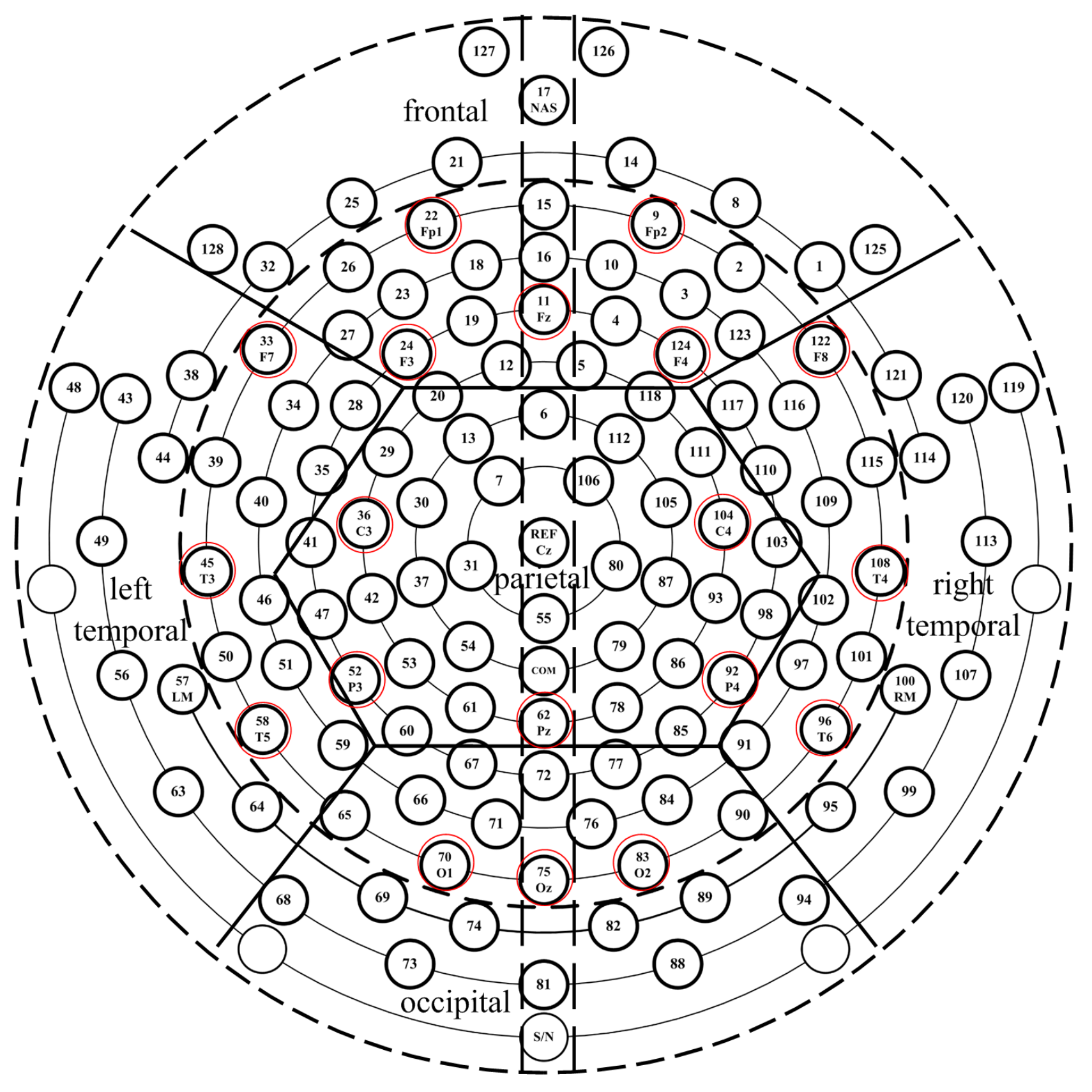




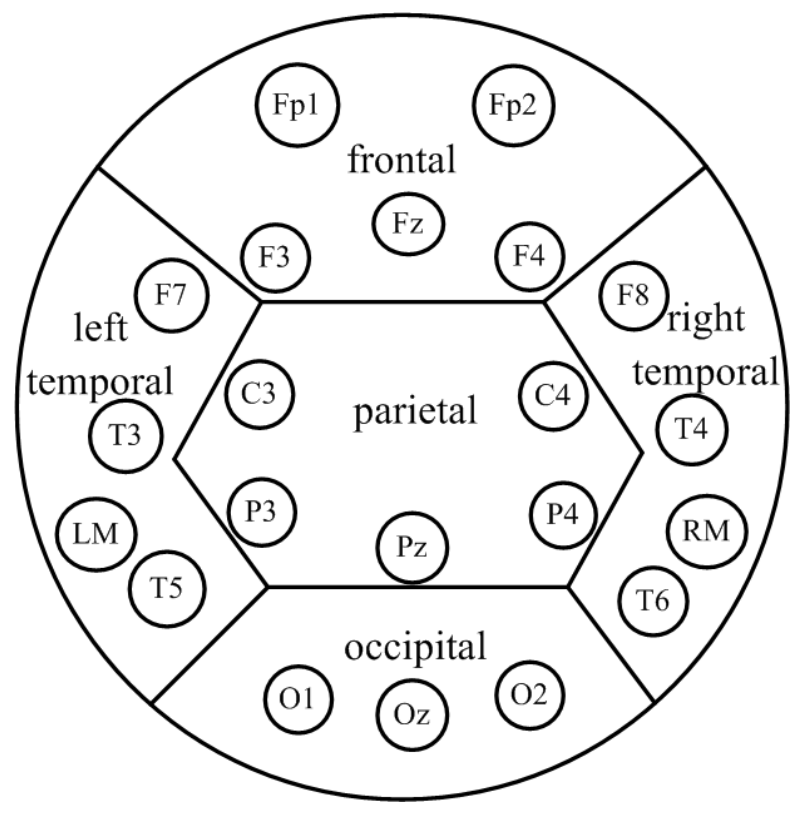




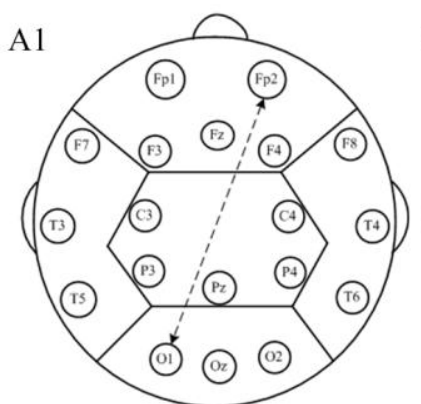

A2 $0.031 \pm 0.017 \quad 0.029 \pm 0.015$ vs. vs. $0.049 \pm 0.023 \quad 0.043 \pm 0.021$

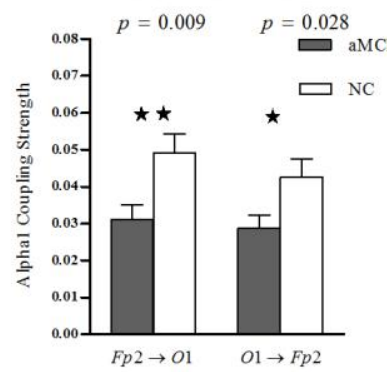

A3

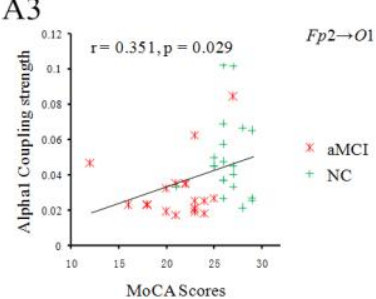

A4

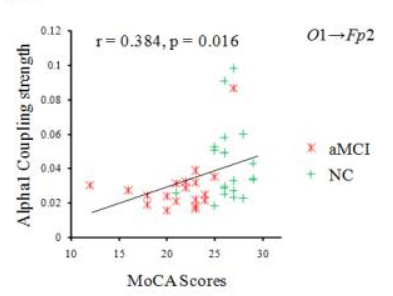

B1

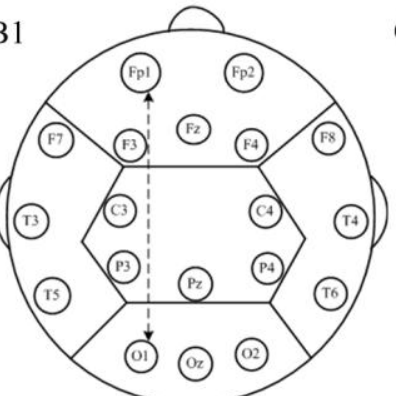

B2 $0.031 \pm 0.016 \quad 0.030 \pm 0.015$ vs. vs. $0.050 \pm 0.023 \quad 0.045 \pm 0.022$

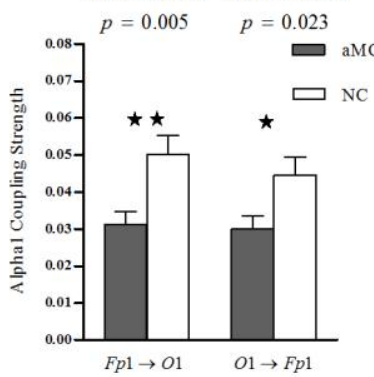

B3

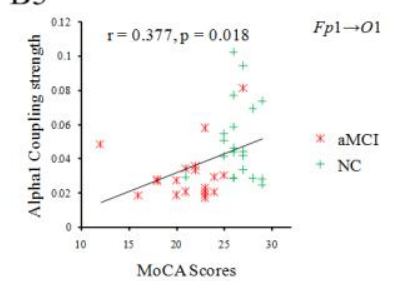

B4

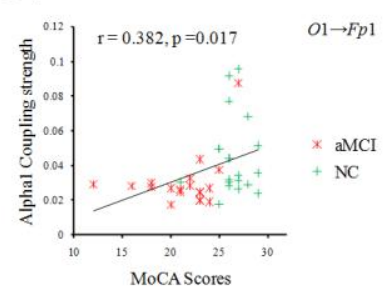

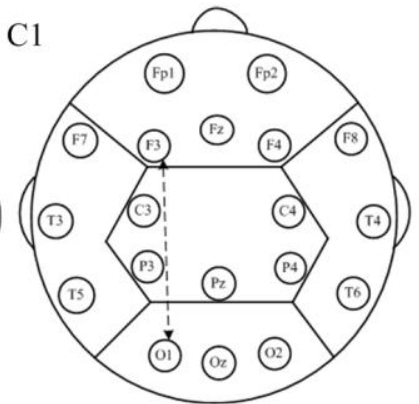

C2 $0.031 \pm 0.016 \quad 0.028 \pm 0.014$ vs. vs.

$0.048 \pm 0.025 \quad 0.044 \pm 0.023$

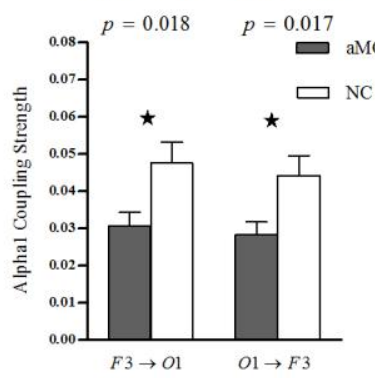

C3

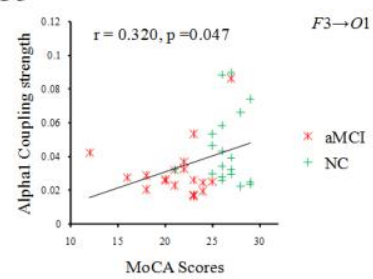

C4

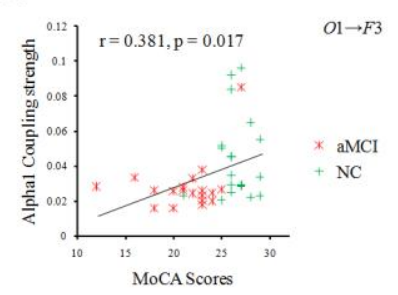

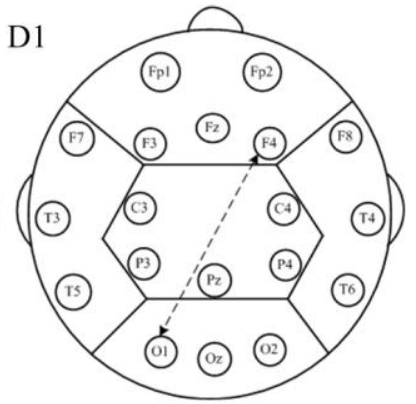

D2 $0.031 \pm 0.017 \quad 0.029 \pm 0.014$ vs. vs. $0.047 \pm 0.023 \quad 0.043 \pm 0.023$

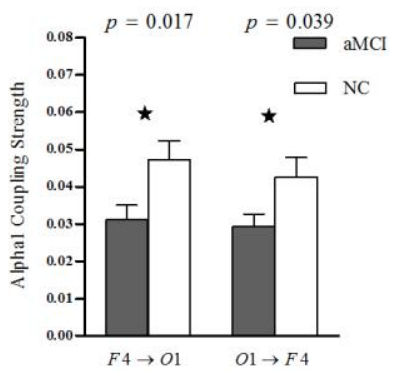

D3

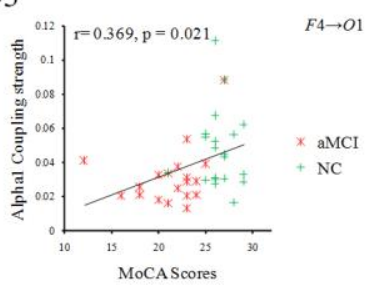

D4

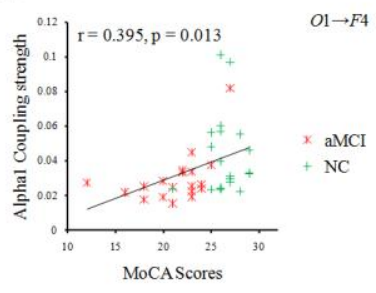


A

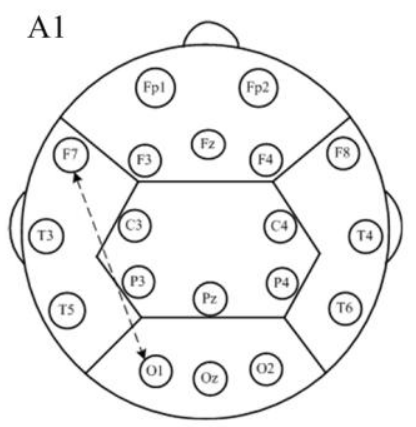

$\begin{array}{ccc}\text { A2 } & 0.031 \pm 0.017 & 0.029 \pm 0.014 \\ \text { vs. } & \text { vs. } \\ 0.047 \pm 0.023 & 0.042 \pm 0.022 \\ p=0.016 & p=0.035\end{array}$

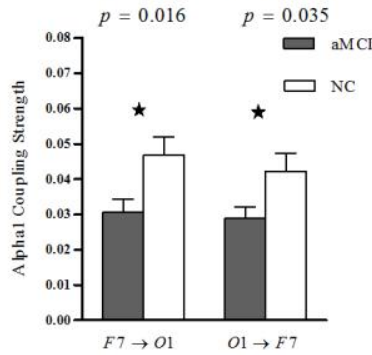

A3
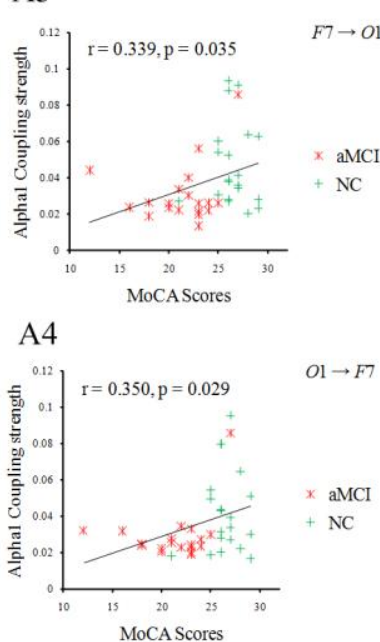

B

B1

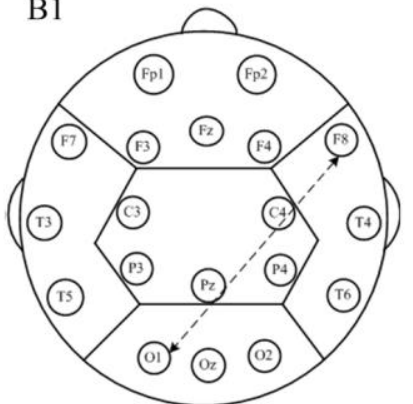

B2 $0.032 \pm 0.016 \quad 0.031 \pm 0.014$

$\begin{array}{cc}\text { vs. } & \text { vs. } \\ 0.049 \pm 0.024 & 0.044 \pm 0.022\end{array}$

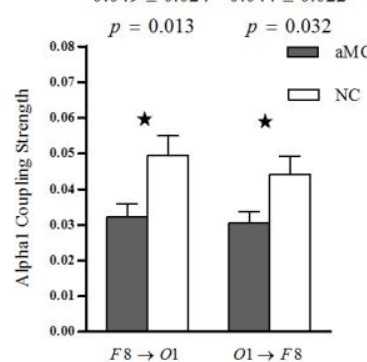

B3

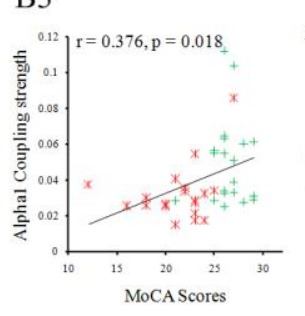

B4

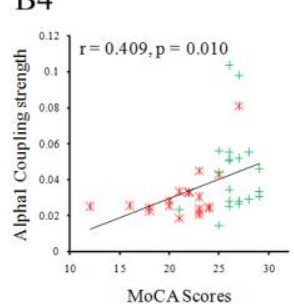

$O 1 \rightarrow F 8$
C

C1

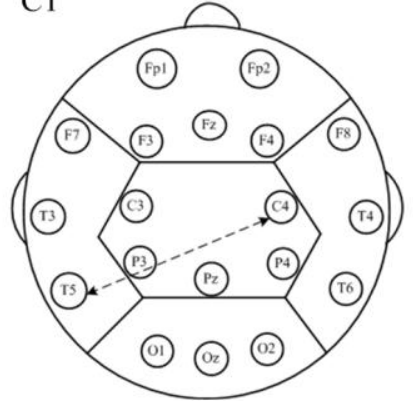

C2 $\quad 0.029 \pm 0.012 \quad 0.027 \pm 0.012$ $\begin{array}{cc}\text { vs. } & \text { vs. } \\ 0.041 \pm 0.021 & 0.039 \pm 0.021\end{array}$

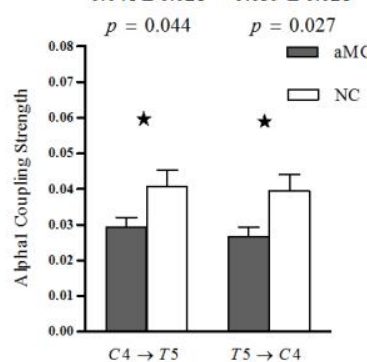

C3

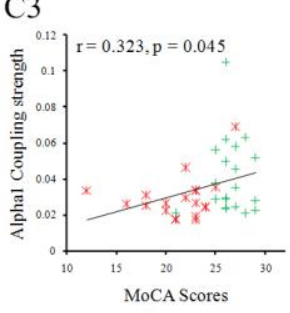

$(4 \rightarrow T 5$

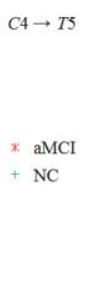

C4

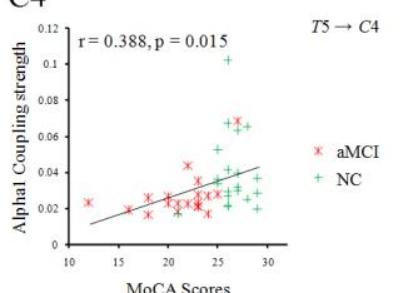

D

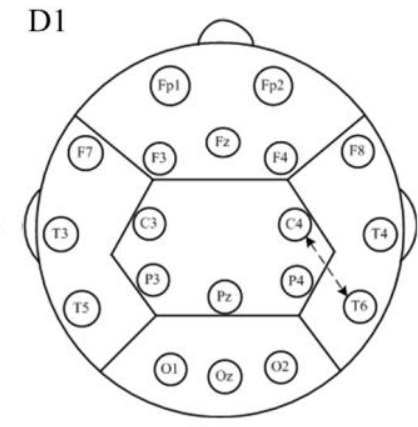

D2 $0.029 \pm 0.009 \quad 0.032 \pm 0.012$ $0.042 \pm 0.016 \quad 0.044 \pm 0.019$

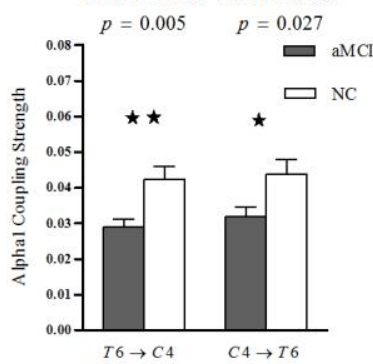

D3

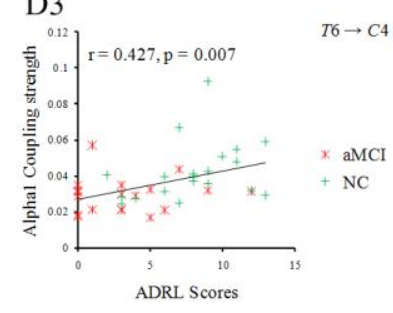

D4

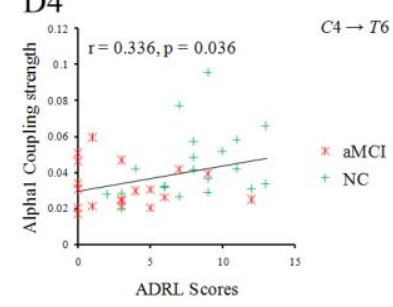




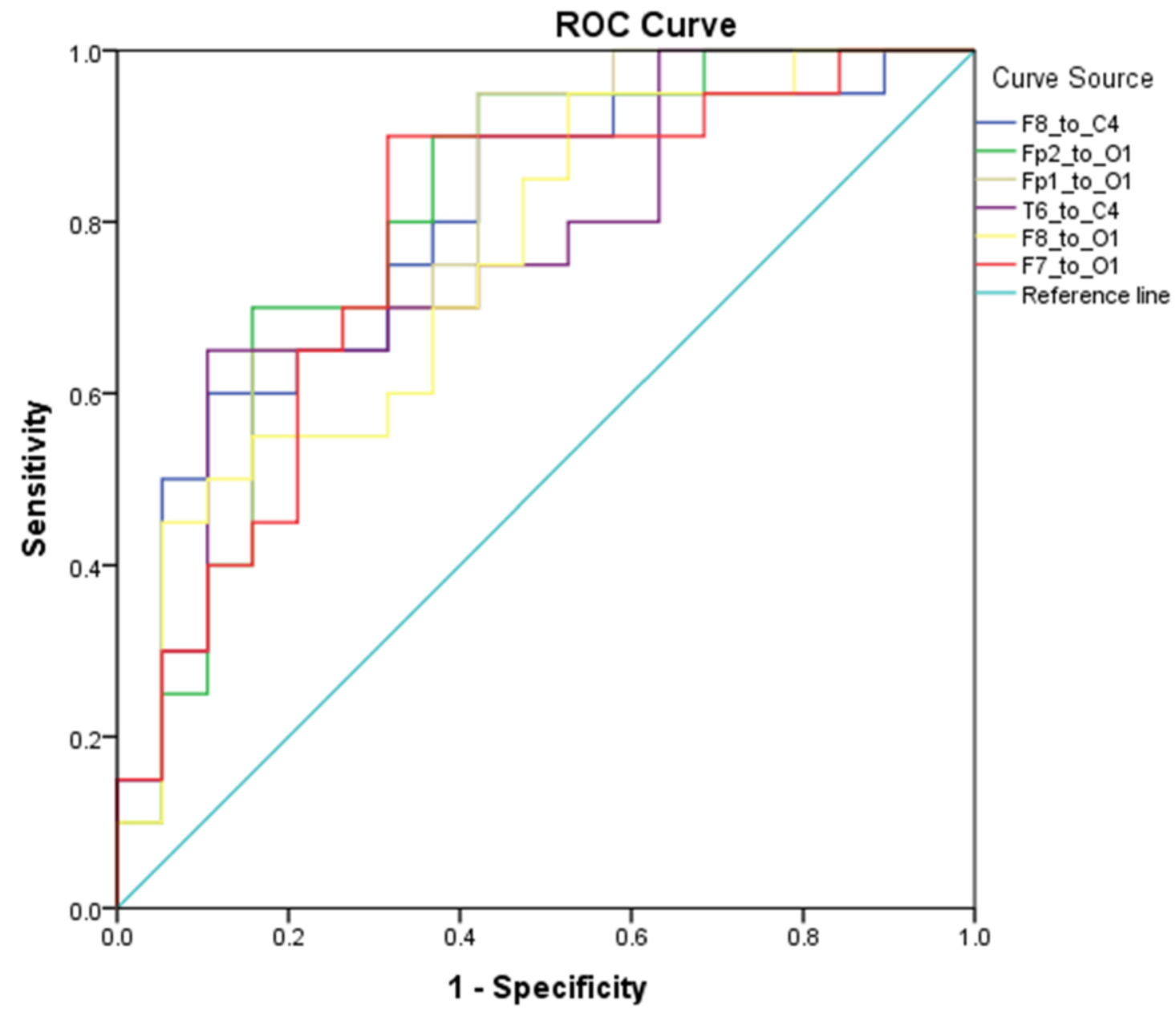



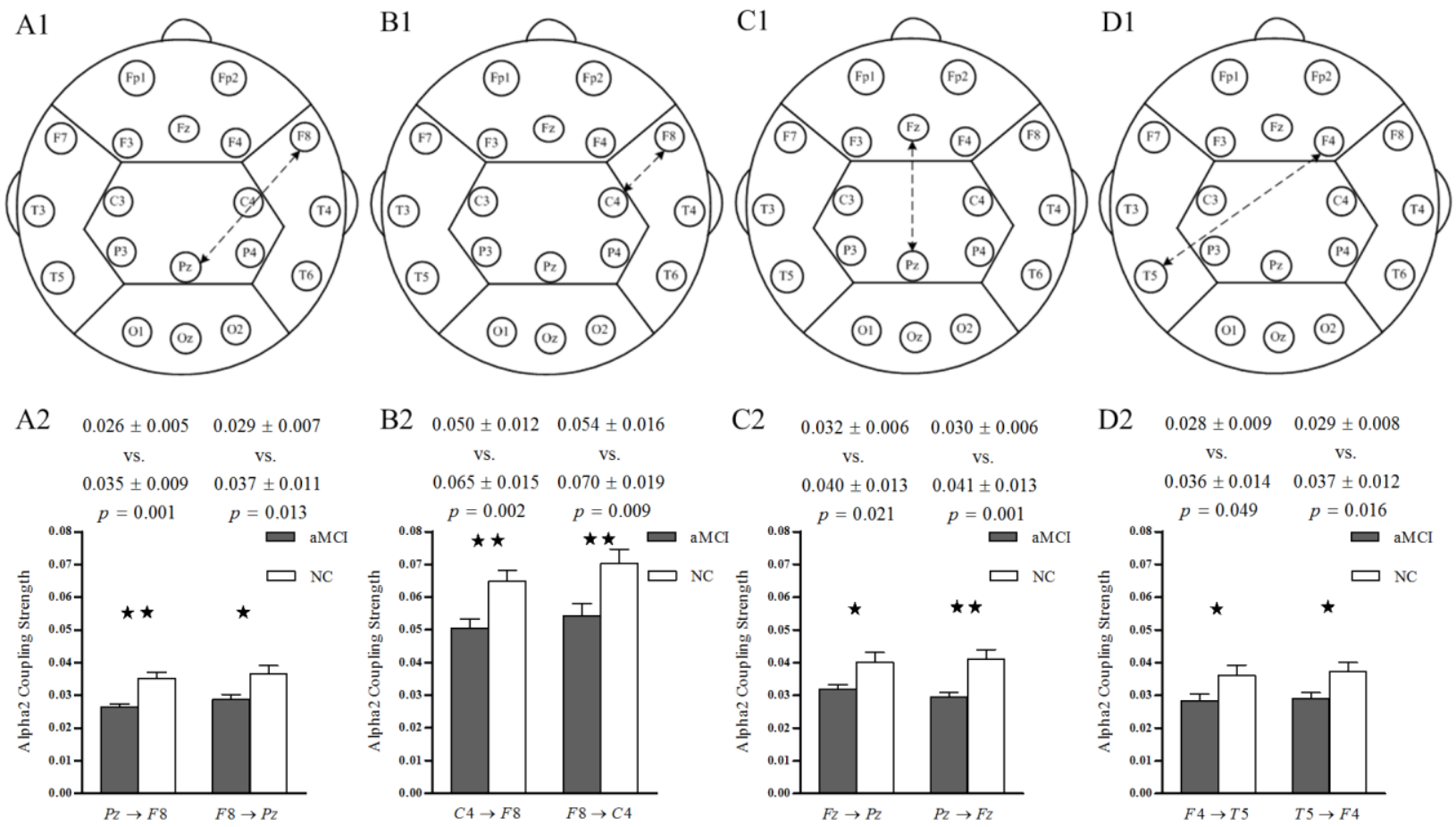

B2 $0.050 \pm 0.0120 .054 \pm 0.016$
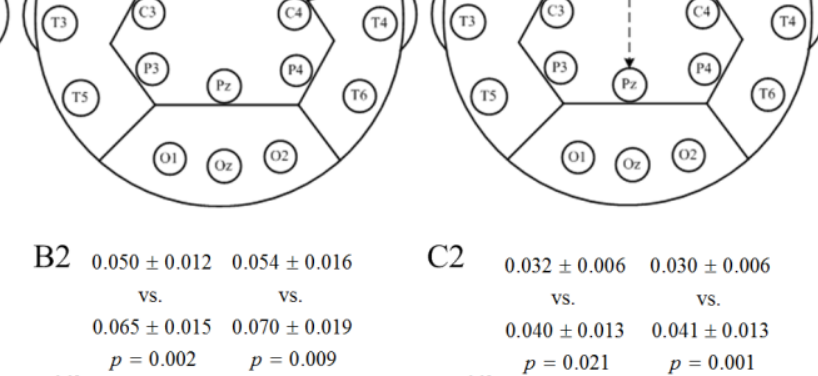

4)

C2 $0.032 \pm 0.006 \quad 0.030 \pm 0.006$

D2 $\quad 0.028 \pm 0.009 \quad 0.029 \pm 0.008$ $\begin{array}{cc}\text { vs. } & \text { vs. } \\ 0.040 \pm 0.013 & 0.041 \pm 0.013\end{array}$

$\begin{array}{cc}\text { vs. } & \text { vs. } \\ 0.036 \pm 0.014 & 0.037 \pm 0.012\end{array}$
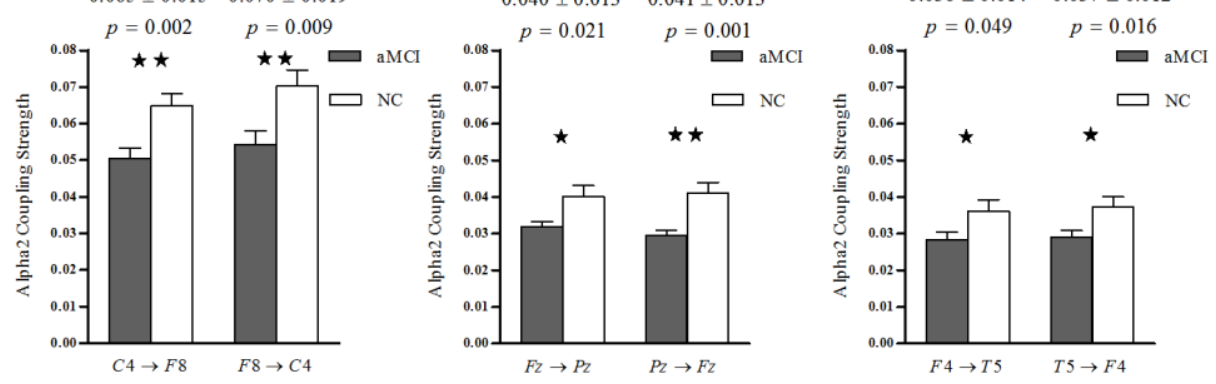

A3

B3

C3

D3
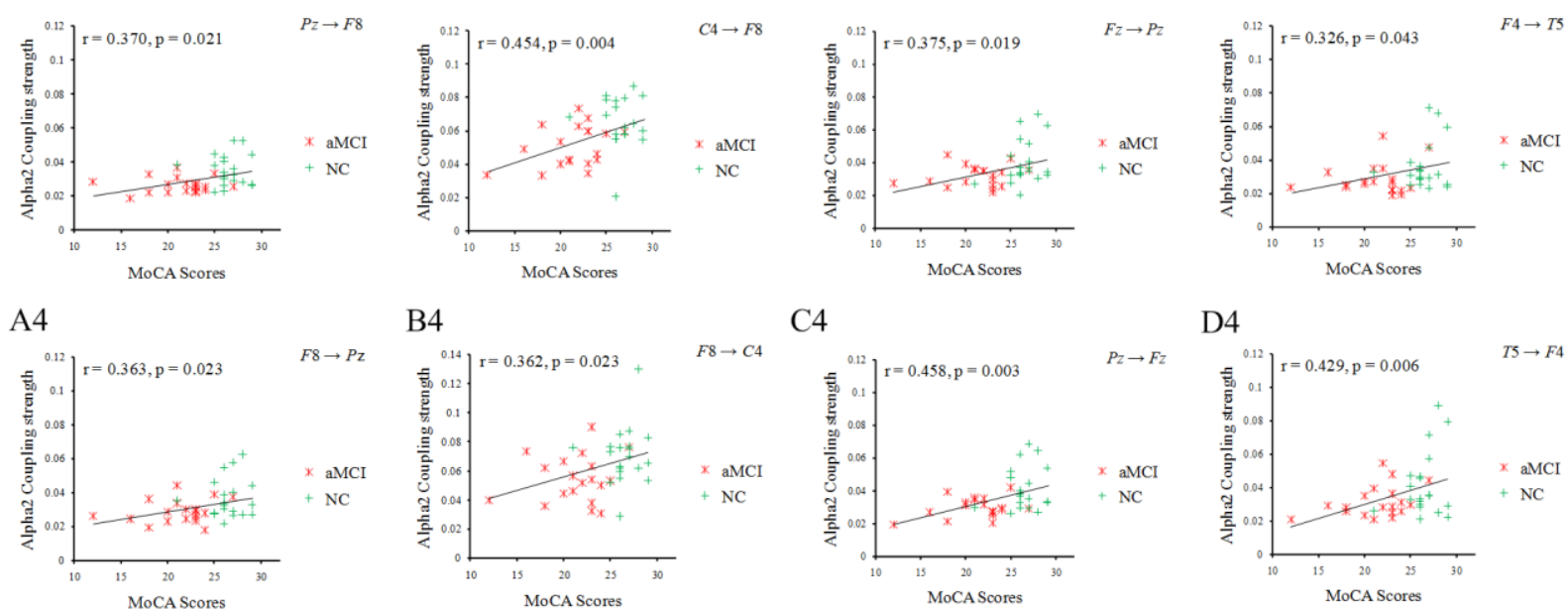

B4

C4
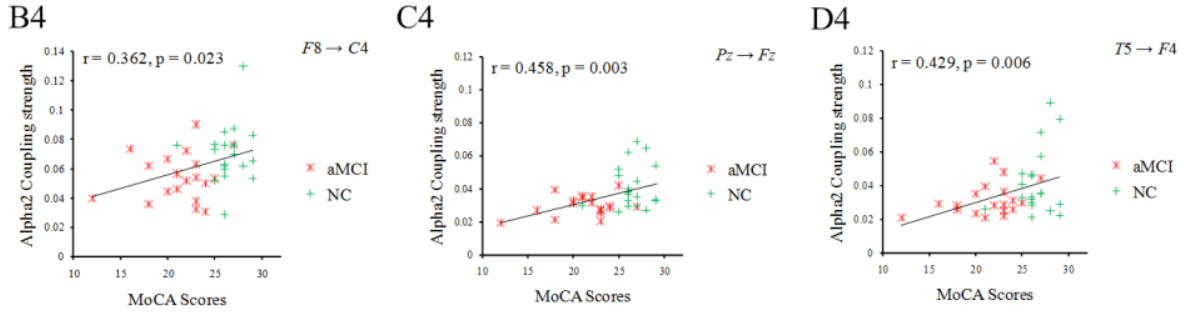


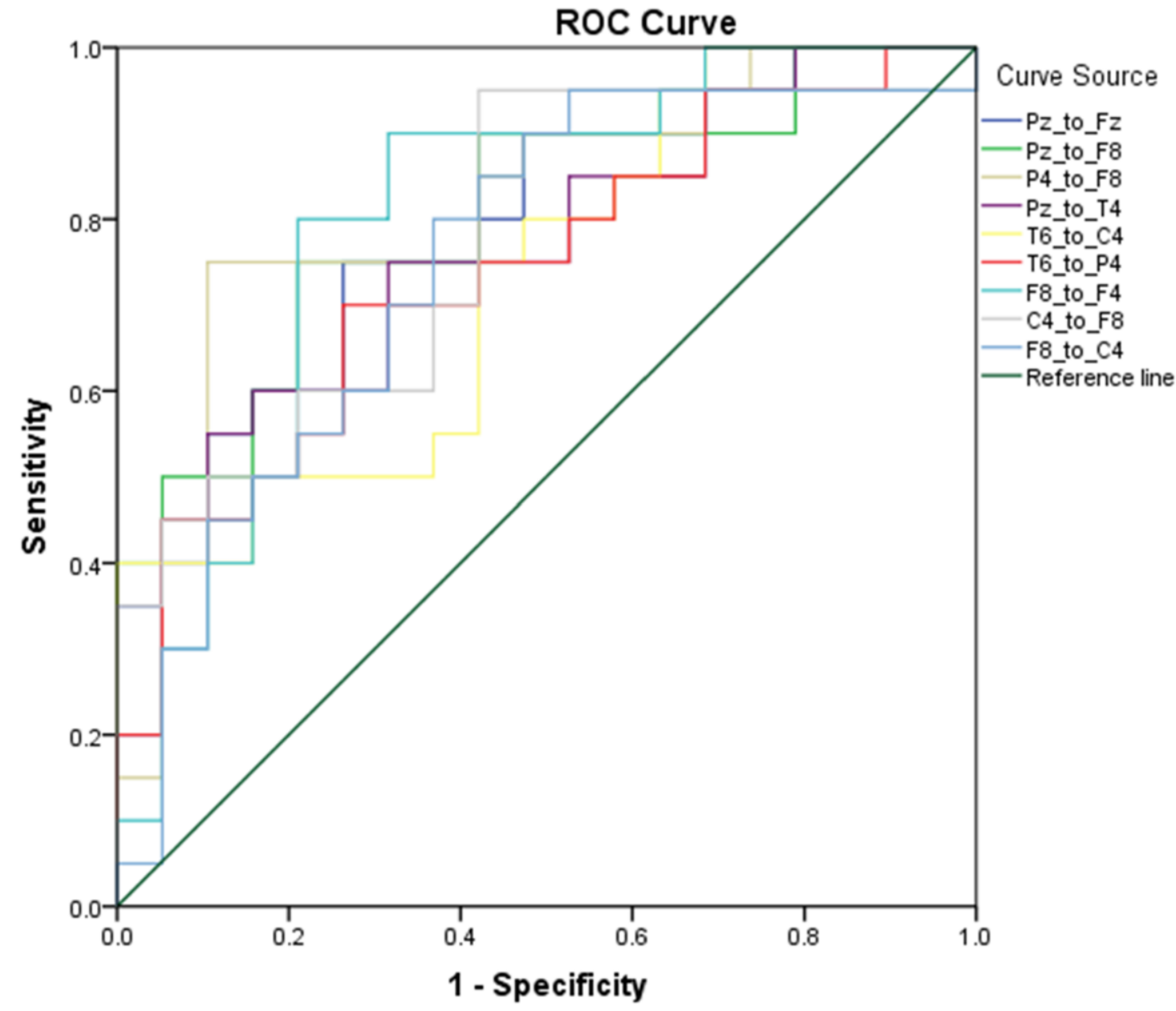




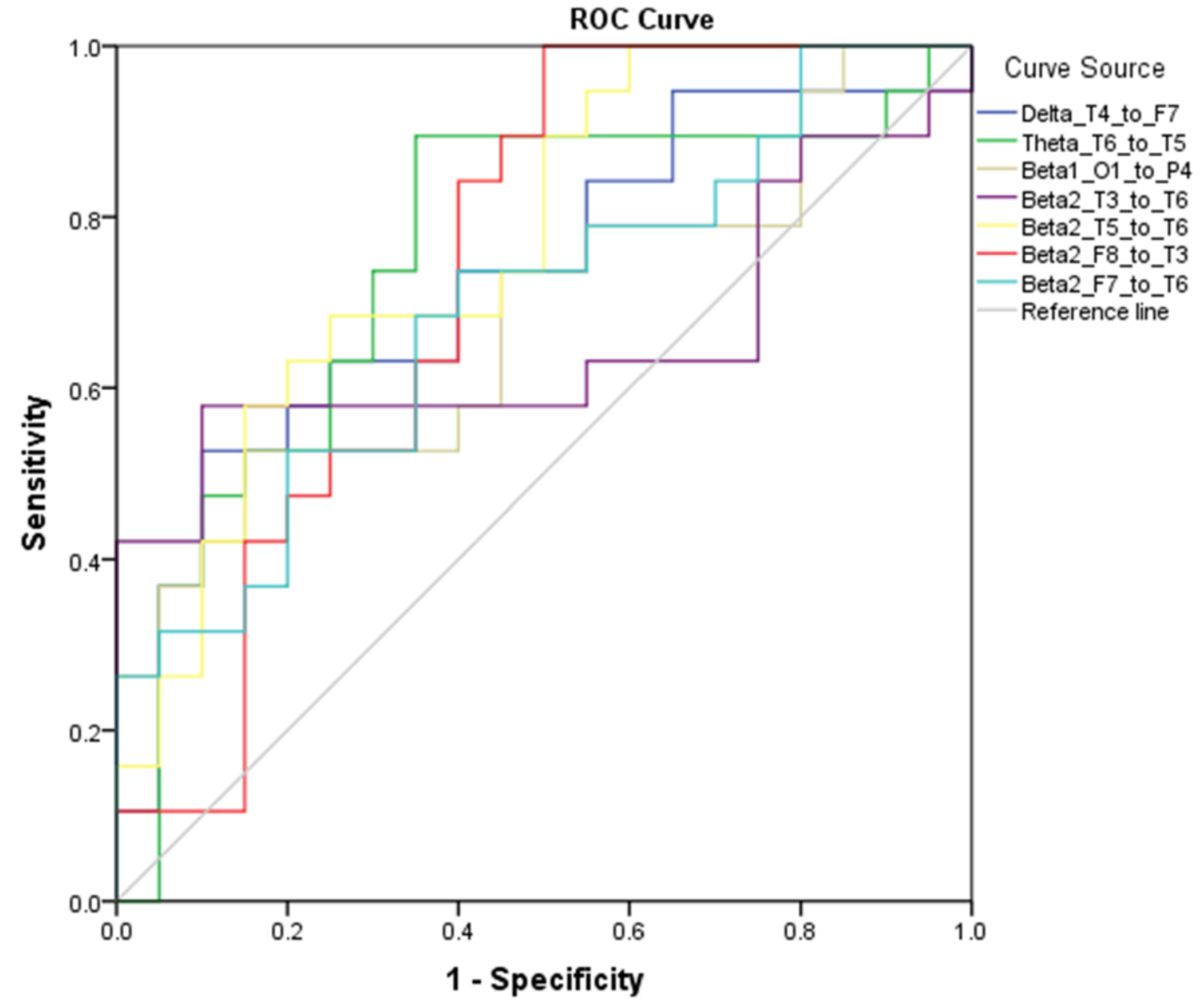


Table 1 Demographic and clinical characteristics:Neuropsychological assessment scores (mean $\pm \mathrm{SEM}), p$ values for the tested items between aMCI and NC groups with T2DM.

\begin{tabular}{|c|c|c|c|}
\hline Items & aMCI (n=19) & $\mathrm{NC}(\mathrm{n}=20)$ & $P$ values \\
\hline Age $(\text { mean } \pm S D, \text { years })^{\mathrm{a}}$ & $68.53 \pm 9.52$ & $69.35 \pm 8.60$ & 0.779 \\
\hline Sex $(\text { Male/Female, } n)^{b}$ & $7 / 12$ & $9 / 11$ & 0.605 \\
\hline Education $\left(\right.$ mean $\pm \mathrm{SD}$, years) ${ }^{\mathrm{a}}$ & $13.00 \pm 2.94$ & $12.70 \pm 3.40$ & 0.770 \\
\hline $\mathrm{MoCA}^{\mathrm{a}}$ & $21.32 \pm 3.46$ & $26.45 \pm 1.82$ & $\mathrm{p}<0.001^{* * *}$ \\
\hline $\operatorname{MMSE}^{\mathrm{a}}$ & $27.37 \pm 2.50$ & $28.55 \pm 1.05$ & 0.06 \\
\hline AVLT-Immediate recall ${ }^{\mathrm{a}}$ & $4.94 \pm 1.79$ & $7.21 \pm 1.78$ & $\mathrm{p}<0.001^{* * *}$ \\
\hline AVLT- Delayed recall $^{\mathrm{a}}$ & $3.26 \pm 3.43$ & $7.95 \pm 3.27$ & $\mathrm{p}<0.001^{* * *}$ \\
\hline AVLT-Delayed recognition ${ }^{\mathrm{a}}$ & $10.21 \pm 4.25$ & $13.25 \pm 1.55$ & $0.008^{* *}$ \\
\hline Boston Name Test ${ }^{\mathrm{a}}$ & $18.61 \pm 1.50$ & $19.80 \pm 0.41$ & $0.004^{* *}$ \\
\hline Trail Making Test $1^{\text {a }}$ & $66.72 \pm 20.44$ & $55.74 \pm 17.78$ & 0.087 \\
\hline Trail Making Test $2^{\mathrm{a}}$ & $113.78 \pm 49.28$ & $106.78 \pm 38.26$ & 0.637 \\
\hline Verbal Fluency Test ${ }^{\mathrm{a}}$ & $14.84 \pm 3.00$ & $18.75 \pm 4.05$ & $0.002^{* *}$ \\
\hline WAIS-DST $^{\mathrm{a}}$ & $11.37 \pm 2.59$ & $13.95 \pm 3.08$ & $0.008^{* * *}$ \\
\hline Activity of Daily Living Scale ${ }^{a}$ & $2.21 \pm 3.17$ & $0.26 \pm 1.14$ & $0.019^{*}$ \\
\hline
\end{tabular}

a:Independent sample $\mathrm{t}$ test; $\mathrm{b}$ : Chi-square test

$*$ indicate $p<0.05$ (significant), $* *$ and $* * * \quad$ indicate $p<0.01$ (extremely significant)

Table 2. The mean and standard deviation of coupling strength, correlation coefficient $\left(r_{1}, r_{2}\right)$ between the strength values and different neuropsychological assessment scores, and their significance $\left(p_{0}, p_{1}, p_{2}\right)$ from two different brain region of aMCI and NC on Alpha1 frequency band.

\begin{tabular}{|c|c|c|c|c|c|c|}
\hline Brain regions & $\begin{array}{c}\text { Electrode } \\
\text { pair }\end{array}$ & $\begin{array}{c}\mathrm{aMCI} \\
(\mathrm{mean} \pm \mathrm{SD})\end{array}$ & $\begin{array}{c}\mathrm{NC} \\
(\mathrm{mean} \pm \mathrm{SD})\end{array}$ & $p_{0}$ & $\begin{array}{l}\text { MoCA } \\
\left(r_{1} / p_{1}\right)\end{array}$ & $\begin{array}{c}\text { WAIS-DST } \\
\left(r_{2} / p_{2}\right)\end{array}$ \\
\hline \multirow{4}{*}{ right temporal-parietal } & $F 8 \rightarrow C 4$ & $0.055_{ \pm} 0.016$ & $0.074_{ \pm} 0.019$ & $0.002^{* *}$ & $0.349 / 0.029^{*}$ & $0.374 / 0.021^{*}$ \\
\hline & $F 8 \rightarrow P z$ & $0.034_{ \pm} 0.011$ & $0.050_{ \pm} 0.031$ & $0.041^{*}$ & - & $0.342 / 0.036^{*}$ \\
\hline & $T 4 \rightarrow C 4$ & $0.045 \pm 0.016$ & $0.056 \pm 0.015$ & $0.037^{*}$ & $0.349 / 0.029^{*}$ & - \\
\hline & $T 4 \rightarrow P z$ & $0.028 \pm 0.007$ & $0.039 \pm 0.019$ & $0.023^{*}$ & - & $0.418 / 0.009^{* *}$ \\
\hline \multirow{2}{*}{ parietal-frontal } & $C 4 \rightarrow F 3$ & $0.050_{ \pm} 0.012$ & $0.062 \pm 0.020$ & $0.029^{*}$ & - & $0.347 / 0.033^{*}$ \\
\hline & $F z \rightarrow P z$ & $0.041 \pm 0.015$ & $0.060 \pm 0.039$ & $0.043^{*}$ & - & $0.337 / 0.039^{*}$ \\
\hline right frontal-left temporal & $F 4 \rightarrow T 5$ & $0.030_{ \pm} 0.012$ & $0.040 \pm 0.016$ & $0.029^{*}$ & $0.391 / 0.014^{*}$ & - \\
\hline
\end{tabular}

$*$ and ${ }^{* *}$ indicate $p<0.05$ (significant) and $p<0.01$ (extremely significant) respectively. 
Table 3. The accuracy, sensitivity, specificity of classification and AUC from different electrode pairs on Alpha1 frequency band

\begin{tabular}{lllll}
\hline Electrode pairs & Accuracy & Sensitivity & Specificity & AUC \\
\hline$F 8 \rightarrow C 4$ & $71.79 \%$ & $68.42 \%$ & $75 \%$ & 0.795 \\
$F p 2 \rightarrow O 1$ & $71.80 \%$ & $63.16 \%$ & $80 \%$ & 0.805 \\
$F p 1 \rightarrow O 1$ & $74.36 \%$ & $84.21 \%$ & $65 \%$ & 0.795 \\
$T 6 \rightarrow C 4$ & $74.79 \%$ & $78.95 \%$ & $65 \%$ & 0.766 \\
$F 8 \rightarrow O 1$ & $69.23 \%$ & $89.47 \%$ & $50 \%$ & 0.755 \\
$F 7 \rightarrow O 1$ & $69.24 \%$ & $68.42 \%$ & $70 \%$ & 0.779 \\
Above all pairs & $92.31 \%$ & $89.47 \%$ & $95 \%$ & - \\
\hline
\end{tabular}

Table 4.The mean and standard deviation of coupling strength, correlation coefficient $(r)$ between the strength values and MoCA scores, and their significance $\left(p_{1}, p_{2}\right)$ from two different brain region in Alpha2 frequency

\begin{tabular}{lccccc}
\hline \multirow{2}{*}{ Brain regions } & $\begin{array}{c}\text { Electrode } \\
\text { pair }\end{array}$ & $\begin{array}{c}\text { aMCI } \\
(\text { mean } \pm \text { SD })\end{array}$ & $\begin{array}{c}\text { NC } \\
(\text { mean } \pm \mathrm{SD})\end{array}$ & $p_{1}$ & $\begin{array}{c}\text { MoCA } \\
\left(r / p_{2}\right)\end{array}$ \\
\hline & $T 6 \rightarrow C 4$ & $0.032_{ \pm} 0.006$ & $0.044_{ \pm} 0.017$ & $0.007^{* *}$ & $0.375 / 0.019^{*}$ \\
& $T 6 \rightarrow P 4$ & $0.057_{ \pm} 0.013$ & $0.072_{ \pm} 0.021$ & $0.009^{* *}$ & $0.328 / 0.041^{*}$ \\
right temporal-parietal & $T 4 \rightarrow C 4$ & $0.044_{ \pm} 0.012$ & $0.054_{ \pm} 0.014$ & $0.019^{*}$ & $0.416 / 0.008^{* *}$ \\
& $F 8 \rightarrow C 3$ & $0.035_{ \pm} 0.012$ & $0.045_{ \pm} 0.017$ & $0.046^{*}$ & $0.348 / 0.030^{*}$ \\
& $P z \rightarrow T 4$ & $0.026_{ \pm} 0.005$ & $0.032_{ \pm} 0.007$ & $0.003^{* *}$ & $0.354 / 0.027^{*}$ \\
left occipital-frontal & $P 4 \rightarrow F 8$ & $0.034_{ \pm} 0.008$ & $0.045_{ \pm} 0.011$ & $0.001^{* *}$ & $0.426 / 0.007^{* *}$ \\
\hline occipital- right temporal & $O 1 \rightarrow F p 1$ & $0.029_{ \pm} 0.010$ & $0.041_{ \pm} 0.021$ & $0.040^{*}$ & $0.364 / 0.023^{*}$ \\
& $O 1 \rightarrow F 3$ & $0.031_{ \pm} 0.009$ & $0.041_{ \pm} 0.017$ & $0.024^{*}$ & $0.380 / 0.017^{*}$ \\
\hline right temporal- right frontal & $F 8 \rightarrow F 4$ & $0.064_{ \pm} 0.010$ & $0.074_{ \pm} 0.009$ & $0.002^{* *}$ & $0.347 / 0.031^{*}$ \\
\hline left temporal- right occipital & $T 3 \rightarrow O 2$ & $0.029_{ \pm} 0.008$ & $0.037_{ \pm} 0.014$ & $0.041^{*}$ & $0.327 / 0.042^{*}$ \\
\hline
\end{tabular}

* and ${ }^{* *}$ indicate $p<0.05$ (significant) and $p<0.01$ (extremely significant) respectively 
Table 5. The accuracy, sensitivity, specificity of classification and AUC from different electrode pairs on Alpha2 frequency band

\begin{tabular}{llllll}
\hline label & Electrode pairs & Accuracy & Sensitivity & Specificity & AUC \\
\hline 1 & $P z \rightarrow F z$ & $66.67 \%$ & $57.89 \%$ & $75 \%$ & 0.792 \\
2 & $P z \rightarrow F 8$ & $71.79 \%$ & $78.95 \%$ & $65 \%$ & 0.805 \\
3 & $P 4 \rightarrow F 8$ & $79.49 \%$ & $84.21 \%$ & $75 \%$ & 0.813 \\
4 & $P z \rightarrow T 4$ & $66.67 \%$ & $89.47 \%$ & $45 \%$ & 0.761 \\
5 & $T 6 \rightarrow C 4$ & $66.67 \%$ & $36.84 \%$ & $95 \%$ & 0.732 \\
6 & $T 6 \rightarrow P 4$ & $64.10 \%$ & $47.37 \%$ & $80 \%$ & 0.739 \\
7 & $F 8 \rightarrow F 4$ & $71.79 \%$ & $78.95 \%$ & $65 \%$ & 0.803 \\
8 & $C 4 \rightarrow F 8$ & $74.36 \%$ & $52.63 \%$ & $95 \%$ & 0.776 \\
9 & $F 8 \rightarrow C 4$ & $71.79 \%$ & $57.89 \%$ & $85 \%$ & 0.750 \\
10 & $1,4,5,6,7,8$, and 9 pairs & $92.31 \%$ & $89.47 \%$ & $95 \%$ & - \\
11 & $2,4,5,6,7,8$, and 9 pairs & $97.44 \%$ & $94.74 \%$ & $100 \%$ & - \\
12 & $3,4,5,6,7,8$, and 9 pairs & $100 \%$ & $100 \%$ & $100 \%$ & - \\
\hline
\end{tabular}


Table 6.The mean and standard deviation of coupling strength, correlation coefficient $\left(r_{1}, r_{2}, r_{3}, r_{4}, r_{5}\right)$ between the strength values and different neuropsychological assessment scores, and their significance $\left(p_{0}, p_{1}, p_{2}, p_{3}, p_{4}, p_{5}\right)$ from two different brain region of aMCI and NC on Delta, Theta, Beta1 and Beta2 frequency bands.

\begin{tabular}{|c|c|c|c|c|c|c|c|c|c|c|}
\hline $\begin{array}{c}\text { Frequency } \\
\text { band }\end{array}$ & Brain regions & $\begin{array}{c}\text { Electrode } \\
\text { pair }\end{array}$ & $\begin{array}{c}\text { aMCI } \\
\text { (mean } \pm \\
\text { SD) }\end{array}$ & $\begin{array}{c}\mathrm{NC} \\
(\text { mean } \pm \\
\mathrm{SD})\end{array}$ & $p_{0}$ & $\begin{array}{l}\text { MoCA } \\
\left(r_{1} / p_{1}\right)\end{array}$ & $\begin{array}{l}\mathrm{ADRL} \\
\left(r_{2} / p_{2}\right)\end{array}$ & $\begin{array}{l}\text { ADRN } \\
\left(r_{3} / p_{3}\right)\end{array}$ & $\begin{array}{l}\mathrm{BNT} \\
\left(r_{4} / p_{4}\right)\end{array}$ & $\begin{array}{l}\text { VFT } \\
\left(r_{5} / p_{5}\right)\end{array}$ \\
\hline Delta & $\begin{array}{l}\text { right temporal } \\
\text {-left temporal }\end{array}$ & $T 4 \rightarrow F 7$ & $\begin{array}{c}0.020 \\
\pm 0.006\end{array}$ & $\begin{array}{c}0.019 \\
\pm 0.004\end{array}$ & $0.020^{*}$ & - & - & - & $\begin{array}{l}0.498 / \\
0.001^{* *}\end{array}$ & - \\
\hline \multirow{2}{*}{ Theta } & $\begin{array}{l}\text { right temporal } \\
\text {-left temporal }\end{array}$ & $T 6 \rightarrow T 5$ & $\begin{array}{c}0.036 \\
\pm 0.009\end{array}$ & $\begin{array}{c}0.031 \\
\pm 0.008\end{array}$ & $0.038^{*}$ & $\begin{array}{l}-0.347 / \\
0.031^{*}\end{array}$ & - & - & - & - \\
\hline & $\begin{array}{l}\text { right temporal } \\
\text {-right frontal }\end{array}$ & $F 8 \rightarrow F 4$ & $\begin{array}{c}0.064 \\
\pm 0.008\end{array}$ & $\begin{array}{c}0.069 \\
\pm 0.008\end{array}$ & $0.048^{*}$ & $\begin{array}{l}0.328 / \\
0.041^{*}\end{array}$ & - & - & - & - \\
\hline \multirow{3}{*}{ Beta1 } & right temporal & $T 6 \rightarrow T 3$ & $\begin{array}{c}0.045 \\
\pm 0.018\end{array}$ & $\begin{array}{c}0.034 \\
\pm 0.008\end{array}$ & $0.033^{*}$ & - & $\begin{array}{l}-0.356 / \\
0.026^{*}\end{array}$ & - & - & - \\
\hline & -left temporal & $F 8 \rightarrow T 5$ & $\begin{array}{c}0.034 \\
\pm 0.009\end{array}$ & $\begin{array}{c}0.029 \\
\pm 0.004\end{array}$ & $0.034^{*}$ & - & - & - & - & $\begin{array}{l}-0.338 / \\
0.035^{*}\end{array}$ \\
\hline & $\begin{array}{l}\text { left occipital } \\
\text {-right parietal }\end{array}$ & $O 1 \rightarrow P 4$ & $\begin{array}{c}0.032 \\
\pm 0.006\end{array}$ & $\begin{array}{c}0.028 \\
\pm 0.003\end{array}$ & $0.023^{*}$ & $\begin{array}{l}-0.320 / \\
0.047^{*}\end{array}$ & - & - & - & - \\
\hline \multirow{7}{*}{ Beta2 } & \multirow{6}{*}{$\begin{array}{l}\text { left temporal } \\
\text {-right temporal }\end{array}$} & $F 7 \rightarrow T 6$ & $\begin{array}{c}0.036 \\
\pm 0.012\end{array}$ & $\begin{array}{c}0.028 \\
\pm 0.004\end{array}$ & $0.016^{*}$ & - & $\begin{array}{l}-0.337 / \\
0.036^{*}\end{array}$ & - & - & - \\
\hline & & $T 6 \rightarrow F 7$ & $\begin{array}{c}0.034 \\
\pm 0.007\end{array}$ & $\begin{array}{c}0.029 \\
\pm 0.004\end{array}$ & $0.020^{*}$ & - & $\begin{array}{l}-0.371 / \\
0.020^{*}\end{array}$ & - & - & - \\
\hline & & $T 3 \rightarrow T 6$ & $\begin{array}{c}0.039 \\
\pm 0.014\end{array}$ & $\begin{array}{c}0.030 \\
\pm 0.005\end{array}$ & $0.009^{* * *}$ & - & $\begin{array}{l}-0.344 / \\
0.032^{*}\end{array}$ & - & - & - \\
\hline & & $T 6 \rightarrow T 3$ & $\begin{array}{c}0.038 \\
\pm 0.011\end{array}$ & $\begin{array}{c}0.031 \\
\pm 0.005\end{array}$ & $0.028^{*}$ & - & $\begin{array}{l}-0.355 / \\
0.026^{*}\end{array}$ & - & - & - \\
\hline & & $T 5 \rightarrow T 6$ & $\begin{array}{c}0.034 \\
\pm 0.008\end{array}$ & $\begin{array}{c}0.029 \\
\pm 0.004\end{array}$ & $0.011^{*}$ & - & $\begin{array}{l}-0.384 / \\
0.016^{*}\end{array}$ & $\begin{array}{l}-0.521 / \\
0.001^{* *}\end{array}$ & - & - \\
\hline & & $F 8 \rightarrow T 3$ & $\begin{array}{c}0.031 \\
\pm 0.005\end{array}$ & $\begin{array}{c}0.027 \\
\pm 0.005\end{array}$ & $0.013^{*}$ & - & - & $\begin{array}{l}-0.374 / \\
0.019^{*}\end{array}$ & 一 & - \\
\hline & $\begin{array}{l}\text { right frontal } \\
\text {-left frontal }\end{array}$ & $F 4 \rightarrow F p 1$ & $\begin{array}{c}0.055 \\
\pm 0.013\end{array}$ & $\begin{array}{c}0.047 \\
\pm 0.008\end{array}$ & $0.048^{*}$ & - & $\begin{array}{l}-0.350 / \\
0.029^{*}\end{array}$ & - & - & - \\
\hline
\end{tabular}

Abbreviations: MoCA, Montreal Cognitive Assessment; ADRL, Auditory Verbal Learning Test- Delayed recall; ADRN,Auditory Verbal Learning Test-Delayed recognition; BNT, Boston Naming Test; VFT, Verbal Fluency Test.

$*$ and $* *$ indicate $p<0.05$ (significant) and $p<0.01$ (extremely significant) respectively 
Table 7. The accuracy, sensitivity, specificity of classification and AUC from different electrode pairs on multiple frequency bands

\begin{tabular}{lllllll}
\hline label & Frequency bands & Electrode pairs & Accuracy & Sensitivity & Specificity & AUC \\
\hline 1 & Delta & $T 4 \rightarrow F 7$ & $64.10 \%$ & $94.74 \%$ & $35 \%$ & 0.726 \\
2 & Theta & $T 6 \rightarrow T 5$ & $74.36 \%$ & $84.21 \%$ & $65 \%$ & 0.753 \\
3 & Beta1 & $O 1 \rightarrow P 4$ & $69.23 \%$ & $52.63 \%$ & $85 \%$ & 0.682 \\
4 & Beta2 & $T 3 \rightarrow T 6$ & $71.79 \%$ & $57.89 \%$ & $85 \%$ & 0.653 \\
5 & Beta2 & $T 5 \rightarrow T 6$ & $64.10 \%$ & $73.68 \%$ & $55 \%$ & 0.768 \\
6 & Beta2 & $F 8 \rightarrow T 3$ & $64.10 \%$ & $94.74 \%$ & $35 \%$ & 0.732 \\
7 & Beta2 & $F 7 \rightarrow T 6$ & $64.10 \%$ & $63.16 \%$ & $65 \%$ & 0.692 \\
8 & Beta2 & $4,5,6$, and 7 pairs & $92.31 \%$ & $94.74 \%$ & $90 \%$ & - \\
\hline
\end{tabular}

Table 8. The number of forward direction and backward direction between two electrodes from two brain regions, correlation coefficient $\left(r_{1}, r_{2}, r_{3}\right)$ between the directionality indexes and different neuropsychological assessment scores, and their different and correlation significance $\left(p_{0}, p_{1}, p_{2}, p_{3}\right)$ of aMCI and NC in Alpha1 frequency.

\begin{tabular}{|c|c|c|c|c|c|c|c|}
\hline Brain regions & Electrode pair & $\begin{array}{c}\text { aMCI } \\
\text { (NFD } \\
\text { /NBD) }\end{array}$ & $\begin{array}{c}\mathrm{NC} \\
(\mathrm{NFD} \\
\text { /NBD) }\end{array}$ & $p_{0}$ & $\begin{array}{l}\text { MoCA } \\
\left(r_{1} / p_{1}\right)\end{array}$ & $\begin{array}{l}\text { VFT } \\
\left(r_{2} / p_{2}\right)\end{array}$ & $\begin{array}{l}\text { TMT1 } \\
\left(r_{3} / p_{3}\right)\end{array}$ \\
\hline frontal- & $F p 2 \rightarrow T 3$ & $8 / 11$ & $15 / 5$ & $0.037^{*}$ & $0.410 / 0.009^{* *}$ & - & - \\
\hline to-left temporal & $F z \rightarrow T 3$ & $10 / 9$ & $17 / 3$ & $0.029^{*}$ & $0.455 / 0.004^{* *}$ & - & - \\
\hline $\begin{array}{l}\text { right frontal- } \\
\text { to- left frontal }\end{array}$ & $F p 2 \rightarrow F p 1$ & $13 / 6$ & $7 / 13$ & $0.037^{*}$ & - & $0.448 / 0.004^{* *}$ & - \\
\hline $\begin{array}{l}\text { left frontal- } \\
\text { to-left occipital }\end{array}$ & $F p 1 \rightarrow O 1$ & $8 / 11$ & $17 / 3$ & $0.005^{* *}$ & 一 & - & $0.335 / 0.043^{*}$ \\
\hline $\begin{array}{l}\text { left occipital - } \\
\text { to- right parietal }\end{array}$ & $O 1 \rightarrow P 4$ & $14 / 5$ & $7 / 13$ & $0.015^{*}$ & - & $0.370 / 0.020^{*}$ & - \\
\hline $\begin{array}{l}\text { left parietal- } \\
\text { to- right temporal }\end{array}$ & $C 3 \rightarrow T 4$ & $15 / 4$ & $9 / 11$ & $0.029^{*}$ & - & $0.373 / 0.019^{*}$ & - \\
\hline
\end{tabular}

Abbreviations: NFD, number of forward direction; NBD, number of backward direction; VFT, Verbal Fluency Test; TMT1,Trail Making Test 1 ; and $* *$ indicate $p<0.05$ and $p<0.01$, respectively 
Table 9. The forward direction and backward direction number between two electrodes from two brain regions, correlation coefficient $\left(r_{1}, r_{2}, r_{3}, r_{4}\right)$ between the directionality indexes and different neuropsychological assessment scores, and their different and correlation significance $\left(p_{0}, p_{1}, p_{2}, p_{3}, p_{4}\right)$ of aMCI and NC in Alpha2, Beta1, Delta and Gamma frequency.

\begin{tabular}{|c|c|c|c|c|c|c|c|c|c|}
\hline $\begin{array}{l}\text { Frequency } \\
\text { band }\end{array}$ & Brain regions & $\begin{array}{c}\text { Electrode } \\
\text { pair }\end{array}$ & $\begin{array}{l}\text { aMCI } \\
\text { (NFD } \\
\text { /NBD) }\end{array}$ & $\begin{array}{c}\mathrm{NC} \\
(\mathrm{NFD} \\
\text { /NBD) }\end{array}$ & $p_{0}$ & $\begin{array}{l}\mathrm{MoCA} \\
\left(r_{1} / p_{1}\right)\end{array}$ & $\begin{array}{l}\text { ADRN } \\
\left(r_{2} / p_{2}\right)\end{array}$ & $\begin{array}{c}\mathrm{BNT} \\
\left(r_{3} / p_{3}\right)\end{array}$ & $\begin{array}{c}\text { WD } \\
\left(r_{4} / p_{4}\right)\end{array}$ \\
\hline Delta & $\begin{array}{l}\text { left temporal-to- } \\
\text { right temporal }\end{array}$ & $F 7 \rightarrow F 8$ & $5 / 14$ & $14 / 6$ & $\begin{array}{l}0.006^{*} \\
*\end{array}$ & $\begin{array}{l}0.318 / \\
0.049^{*}\end{array}$ & - & $\begin{array}{l}0.372 / \\
0.024^{*}\end{array}$ & - \\
\hline \multirow{4}{*}{ Alpha2 } & \multirow{2}{*}{$\begin{array}{l}\text { parietal-to- } \\
\text { left temporal }\end{array}$} & $C 3 \rightarrow T 5$ & $11 / 8$ & $4 / 16$ & $0.015^{*}$ & $\begin{array}{l}0.472 / \\
0.002^{* * *}\end{array}$ & - & - & - \\
\hline & & $P 4 \rightarrow T 5$ & $11 / 8$ & $5 / 15$ & $0.037^{*}$ & $\begin{array}{l}0.396 / \\
0.013^{*}\end{array}$ & - & - & - \\
\hline & \multirow{2}{*}{$\begin{array}{l}\text { parietal-to- } \\
\text { right temporal }\end{array}$} & $P z \rightarrow T 4$ & $8 / 11$ & $15 / 5$ & $0.037^{*}$ & - & $\begin{array}{l}0.338 / \\
0.035^{*}\end{array}$ & - & - \\
\hline & & $C 4 \rightarrow T 4$ & $15 / 4$ & $8 / 12$ & $0.013^{*}$ & - & - & $\begin{array}{l}0.364 / \\
0.025^{*}\end{array}$ & - \\
\hline Beta1 & $\begin{array}{l}\text { left temporal-to- } \\
\text { right temporal }\end{array}$ & $T 3 \rightarrow F 8$ & $8 / 11$ & $16 / 4$ & $0.015^{*}$ & - & - & - & $\begin{array}{l}0.379 / \\
0.019^{*}\end{array}$ \\
\hline Gamma & $\begin{array}{l}\text { left frontal-to- } \\
\text { right parietal }\end{array}$ & $F 3 \rightarrow C 4$ & $12 / 7$ & $3 / 17$ & $\begin{array}{l}0.002^{*} \\
*\end{array}$ & $\begin{array}{l}0.387 / \\
0.015^{*}\end{array}$ & - & $\begin{array}{c}0.428 / \\
0.007^{*} \\
*\end{array}$ & - \\
\hline
\end{tabular}

Abbreviations: NFD, number of forward direction; NBD, number of backward direction; ADRN, AVLT-Delayed recognition; BNT, Boston Name Test; WD, WAIS-DST.

$*$ and $* *$ indicate $p<0.05$ (significant) and $p<0.01$ (extremely significant) respectively 
Table 10. The correlation coefficients $\left(r_{1}, r_{2}\right)$ and their significance $\left(p_{1}, p_{2}\right)$ between sex and coupling strength, age and coupling strength of two electrodes from two brain regions of all subjects in Delta, Alpha1, Alpha2, Beta1, Beta2, and Gamma frequency

\begin{tabular}{|c|c|c|c|c|}
\hline Frequency band & Brain regions & Electrode pair & $\operatorname{Sex}\left(r_{1} / p_{1}\right)$ & Age $\left(r_{2} / p_{2}\right)$ \\
\hline Delta & left parietal-right parietal & $C 3 \rightarrow P 4$ & $0.340 / 0.034^{*}$ & - \\
\hline \multirow{5}{*}{ Alpha1 } & parietal-right temporal & $P z \rightarrow T 6$ & $0.408 / 0.010^{* *}$ & - \\
\hline & right temporal-parietal & $T 6 \rightarrow P z$ & $0.336 / 0.036^{*}$ & - \\
\hline & right temporal-right occipital & $T 6 \rightarrow O 2$ & $0.328 / 0.042^{*}$ & - \\
\hline & left temporal-right temporal & $T 5 \rightarrow F 8$ & - & $0.348 / 0.030^{*}$ \\
\hline & right temporal-left temporal & $F 8 \rightarrow T 5$ & - & $0.344 / 0.032^{*}$ \\
\hline \multirow{3}{*}{ Alpha2 } & right occipital-right temporal & $O 2 \rightarrow T 6$ & $0.445 / 0.005^{* *}$ & - \\
\hline & right occipital-right parietal & $O 2 \rightarrow P 4$ & $0.397 / 0.012^{*}$ & - \\
\hline & right parietal-left parietal & $P 4 \rightarrow C 3$ & $0.347 / 0.030^{*}$ & - \\
\hline \multirow{3}{*}{ Beta1 } & right parietal-left temporal & $P 4 \rightarrow T 5$ & - & $-0.322 / 0.046^{*}$ \\
\hline & right parietal-right temporal & $C 4 \rightarrow T 4$ & - & $-0.365 / 0.022^{*}$ \\
\hline & right temporal-right occipital & $T 4 \rightarrow O 2$ & - & $-0.361 / 0.024^{*}$ \\
\hline \multirow{3}{*}{ Beta2 } & left frontal-right frontal & $F 3 \rightarrow F p 2$ & $-0.382 / 0.016^{*}$ & - \\
\hline & left temporal-right temporal & $T 3 \rightarrow T 6$ & $-0.322 / 0.045^{*}$ & - \\
\hline & right temporal-left temporal & $T 6 \rightarrow T 3$ & $-0.333 / 0.038^{*}$ & - \\
\hline \multirow{3}{*}{ Gamma } & right parietal-right occipital & $P 4 \rightarrow O 2$ & $0.372 / 0.020^{*}$ & - \\
\hline & right occipital-left parietal & $\mathrm{O} 2 \rightarrow \mathrm{C} 3$ & $0.442 / 0.005^{* *}$ & - \\
\hline & right occipital-left parietal & $O 2 \rightarrow P 3$ & $0.458 / 0.003^{* *}$ & - \\
\hline
\end{tabular}

$*$ and $* *$ indicate $p<0.05$ (significant) and $p<0.01$ (extremely significant) respectively 
Table 11. The information flow between right parietal and right temporal of subjects of different sex on Alpha2 frequency band

\begin{tabular}{lccc}
\hline & $C 4 \rightarrow T 4$ & $T 4 \rightarrow C 4$ & total on sex \\
\hline man & 6 & 10 & 16 \\
woman & 17 & 6 & 23 \\
total on coupling direction & 23 & 16 & 39 \\
\hline
\end{tabular}

